\title{
The GOME-2 instrument on the Metop series of satellites: instrument design, calibration, and level 1 data processing - an overview
}

\author{
Rosemary Munro ${ }^{1}$, Rüdiger Lang ${ }^{1}$, Dieter Klaes ${ }^{1}$, Gabriele Poli ${ }^{1}$, Christian Retscher ${ }^{1}$, Rasmus Lindstrot ${ }^{1}$, \\ Roger Huckle ${ }^{1}$, Antoine Lacan ${ }^{1}$, Michael Grzegorski ${ }^{1}$, Andriy Holdak ${ }^{1}$, Alexander Kokhanovsky ${ }^{1}$, Jakob Livschitz ${ }^{2}$, \\ and Michael Eisinger ${ }^{3}$ \\ ${ }^{1}$ EUMETSAT, Eumetsat-Allee 1, Darmstadt, Germany \\ ${ }^{2}$ European Space Agency - European Space Research \& Technology Centre (ESA-ESTEC), Noordwijk, the Netherlands \\ ${ }^{3}$ European Space Agency - European Centre for Space Applications and Telecommunications (ESA-ECSAT), Didcot, \\ Oxfordshire, UK
}

Correspondence to: Rosemary Munro (rosemary.munro@eumetsat.int)

Received: 28 April 2015 - Published in Atmos. Meas. Tech. Discuss.: 11 August 2015

Revised: 10 February 2016 - Accepted: 15 February 2016 - Published: 24 March 2016

\begin{abstract}
The Global Ozone Monitoring Experiment-2 (GOME-2) flies on the Metop series of satellites, the space component of the EUMETSAT Polar System. In this paper we will provide an overview of the instrument design, the on-ground calibration and characterization activities, inflight calibration, and level 0 to 1 data processing. The current status of the level 1 data is presented and points of specific relevance to users are highlighted. Long-term level 1 data consistency is also discussed and plans for future work are outlined. The information contained in this paper summarizes a large number of technical reports and related documents containing information that is not currently available in the published literature. These reports and documents are however made available on the EUMETSAT web pages and readers requiring more details than can be provided in this overview paper will find appropriate references at relevant points in the text.
\end{abstract}

\section{The Global Ozone Monitoring Experiment 2 (GOME-2) instrument}

The Global Ozone Monitoring Experiment-2 (GOME-2) (Fig. 1) is an improved version of the Global Ozone Monitoring Experiment on the second European Remote Sensing Satellite (GOME-1/ERS-2) (Munro, 2006). The instrument mass is $73 \mathrm{~kg}$, consumes an average of $58 \mathrm{~W}$ of power, and has a data rate of $400 \mathrm{kbits}^{-1}$. The instrument is mounted on the front side of the satellite bus in the flight direction. For a summary of the instrument and improvements over GOME-1/ERS-2 please see Callies et al. (2000) and Munro (2006). GOME-2 flies on the Metop series of satellites. The Metop satellites form the space component of the EUMETSAT Polar System and fly in a sun-synchronous orbit, at an altitude of approximately $820 \mathrm{~km}$, and with a descending node equator-crossing local solar time of 09:30 LT. The first Metop satellite, Metop-A, was launched on 19 October 2006. After a successful commissioning phase, Metop-B, on 24 April 2013, replaced Metop-A as EUMETSAT's prime operational polar-orbiting satellite. Metop-A and Metop-B now fly in a tandem operations configuration on the same orbit but separated by $48.93 \mathrm{~min}$. Metop-C is currently planned for launch in 2018. For more information about the EUMETSAT Polar System and the Metop series of satellites see Klaes et al. (2007).

GOME-2 is an optical spectrometer fed by a scan mirror which enables across-track scanning in nadir, as well as sideways viewing for polar coverage and instrument characterization measurements using the moon (Callies et al., 2000). This scan mirror can also be directed towards internal calibration sources or towards a diffuser plate for calibration measurements using the sun. GOME-2 senses the Earth's backscattered radiance and extraterrestrial solar irradiance in 


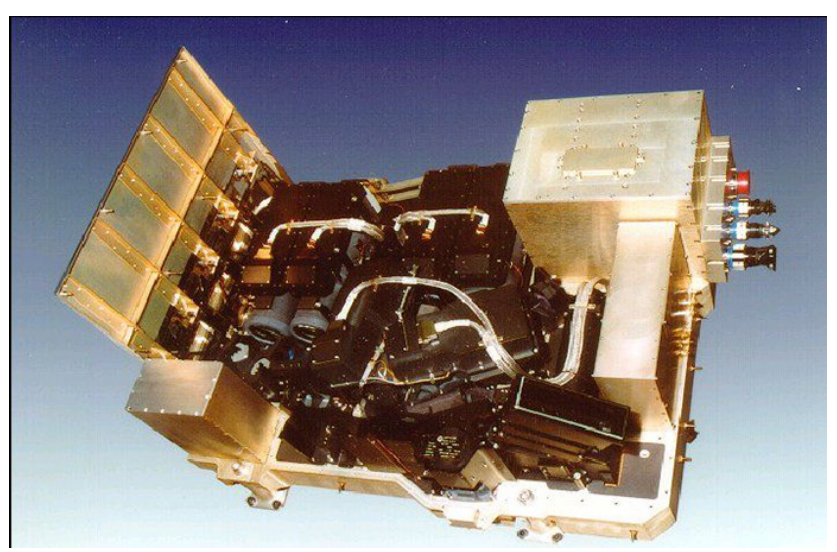

Figure 1. The GOME-2 instrument.

the ultraviolet and visible part of the spectrum (240-790 nm) at a high spectral resolution of $0.26-0.51 \mathrm{~nm}$. The instrument comprises four main optical channels (Focal Plane Assemblies - FPAs) which focus the spectrum on linear detector arrays of 1024 pixels each, and two polarization measurement devices (PMDs) containing the same type of arrays for measurement of linearly polarized intensity in two perpendicular directions. The PMDs are required because GOME-2 is a polarization-sensitive instrument. Therefore, the intensity calibration of GOME-2 has to take account of the polarization state of the incoming light using information from the PMDs. The PMD measurements are performed at lower spectral resolution, but at higher spatial resolution than the main science channels, which also facilitates sub-pixel determination of cloud coverage.

The footprint size is 80 (across-track) $\times 40$ (alongtrack) $\mathrm{km}$ for main science channel data for a nominal swath width of $1920 \mathrm{~km}$. The polarization data are downlinked in 15 spectral bands covering the region from 312 to $800 \mathrm{~nm}$ for both polarization directions with a footprint of 10 (acrosstrack) $\times 40$ (along-track) km, also for a nominal swath width of $1920 \mathrm{~km}$.

The recorded spectra are used to derive a detailed picture of the total atmospheric content of ozone and the vertical ozone profile in the atmosphere. They also provide accurate information on the total column amount of nitrogen dioxide, sulfur dioxide, water vapour, oxygen/oxygen dimer, bromine oxide, and other trace gases, as well as aerosols and cloud optical properties. More information on geophysical product derivation from GOME-2 data can be found in other papers in this issue.

The GOME-2 instrument was developed by Selex Galileo in Florence, Italy, under a joint contract from EUMETSAT and ESA. The characteristics of the main instrument GOME2 are summarized in Table 1.

\subsection{GOME-2 optical layout}

The four main channels (detectors) of the GOME-2 instrument provide continuous spectral coverage of the wavelengths between 240 and $790 \mathrm{~nm}$ with a spectral resolution full width at half maximum (FWHM) between 0.26 and $0.51 \mathrm{~nm}$. Channel characteristics for GOME-2 on Metop-A (Flight Model 3 - FM3) and GOME-2 on Metop-B (Flight Model 2 - FM2) are listed in Table 2. Wavelength values are indicative only and will depend on spectral calibration. Spectral resolution (FWHM) varies slightly across each main channel; the values given are channel averages. In the overlap regions between the main science channels, the wavelengths indicate the $10 \%$ overlap points; e.g. at $310 \mathrm{~nm}, 10 \%$ of the signal is registered in channel 2, and $90 \%$ in channel 1 . At $314 \mathrm{~nm}, 10 \%$ of the signal is registered in channel 1 , and $90 \%$ in channel 2 . Note however that the calibrated level 1 data are generally not fully valid for the whole overlap region. The spectral extent of the valid data is not static, and typically, but not always, extends approximately to the $50 \%$ overlap point, depending on the quality of calibration parameters and corrections. A detailed description of the valid ranges for all operational GOME-2 flight models can be found in EUMETSAT (2014a).

The optical configuration of the instrument is shown in Fig. 2. Light enters the two-mirror telescope system via the scan mirror. The telescope projects the light beam onto the slit, which determines the instantaneous field of view (IFOV) of $0.28^{\circ} \times 2.8^{\circ}$ (across-track $\times$ along-track). After it has passed the slit, the beam is collimated again and enters a double Brewster prism for partial split-off to PMD-S, followed by the pre-disperser prism which has two functions. Brewster reflection at the back of the prism splits off part of the p-polarization direction to PMD-P. The prism furthermore forms a low dispersion spectrum which is subsequently separated at the channel separator prism into three parts going to channels 1 (transmitted beam), 2 (reflected beam), and 3 and 4 (passing the prism edge), respectively. The separation between channels 3 and 4 is performed by a dichroic filter.

A grating in each channel then further disperses the light, which is subsequently focused onto the detector array. The spectral dispersion direction corresponds to the across-track dimension of the slit. Each PMD channel contains a dispersion prism and two additional folding prisms and collimating lenses. PMD-P measures intensity polarized parallel to the spectrometer's slit, and PMD-S measures intensity polarized perpendicular to the spectrometer's slit. The two PMD channels are designed to ensure maximum similarity in their optical properties. The wavelength-dependent dispersion of the prisms causes a much higher spectral resolution in the ultraviolet than in the red part of the spectrum.

In order to reduce the dark signal, the detectors of the main channels are actively cooled by Peltier elements in a closed control loop to temperatures around $235 \mathrm{~K}$. The FPA temperatures for all main channels are very stable due to the closed- 
Table 1. Main GOME-2 instrument characteristics.

\begin{tabular}{|c|c|}
\hline Wavelength range & $240-790 \mathrm{~nm}$ in four main science channels \\
\hline & $\begin{array}{l}300-800 \mathrm{~nm} \text { in two polarization measurement devices (PMDs) } \\
\text { measuring polarization perpendicular to and parallel to the in- } \\
\text { strument slit with } 15 \text { programmable spectral bands }\end{array}$ \\
\hline Spectral sampling & $\begin{array}{l}0.12-0.21 \mathrm{~nm} \text { (main science channels) } \\
0.62-8.8 \text { (PMDs) }\end{array}$ \\
\hline Spectral resolution & $\begin{array}{l}\text { FWHM } 0.26-0.51 \mathrm{~nm} \text { (main science channels) } \\
\text { FWHM } 1.9-55 \mathrm{~nm} \text { ( } 15 \text { programmable PMD spectral bands) }\end{array}$ \\
\hline Orbit & $\begin{array}{l}\text { Sun-synchronous, at an altitude of } \sim 820 \mathrm{~km} \text {, with a descending } \\
\text { node equator-crossing local solar time of 09:30 }\end{array}$ \\
\hline Swath width & Default $1920 \mathrm{~km}$ \\
\hline Spatial resolution & $\begin{array}{l}\text { Default } 80 \mathrm{~km} \text { across-track } \times 40 \mathrm{~km} \text { along-track (main science } \\
\text { channels) and } 10 \mathrm{~km} \text { across-track } \times 40 \mathrm{~km} \text { along-track (PMDs) }\end{array}$ \\
\hline Data rate & $\begin{array}{l}400 \text { kbits s}^{-1} \text { or } 300 \mathrm{MB} \mathrm{orbit}^{-1} \quad \text { (level } 0 \text { data) and } \\
\sim 800 \mathrm{MB} \mathrm{orbit}^{-1} \text { (level 1) }\end{array}$ \\
\hline
\end{tabular}

Table 2. GOME-2 spectral coverage and resolution.

\begin{tabular}{lrrrrr}
\hline Channel & $\begin{array}{r}\text { Approx. spectral } \\
\text { range Metop-A } \\
(\mathrm{nm})\end{array}$ & $\begin{array}{r}\text { Approx. spectral } \\
\text { range Metop-B } \\
(\mathrm{nm})\end{array}$ & $\begin{array}{r}\text { Detector } \\
\text { pixel } \\
\text { size }(\mathrm{nm})\end{array}$ & $\begin{array}{r}\text { FWHM } \\
\text { Metop-A } \\
(\mathrm{nm})\end{array}$ & $\begin{array}{r}\text { FWHM } \\
\text { Metop-B } \\
(\mathrm{nm})\end{array}$ \\
\hline 1 & $240-314$ & $239-312$ & 0.1 & 0.26 & 0.29 \\
2 & $310-403$ & $308-402$ & 0.1 & 0.27 & 0.28 \\
3 & $397-604$ & $395-604$ & 0.2 & 0.51 & 0.55 \\
4 & $593-790$ & $593-791$ & 0.2 & 0.48 & 0.50 \\
PMD-P & & & & & \\
PMD-S & $312-790$ & $312-790$ & $0.62-8.8$ & $2.9-37$ & $2.9-37$ \\
\hline
\end{tabular}

loop cooling configuration. The PMDs are cooled by Peltier elements in an open-loop configuration which reject heat to the main optical bench through the polarization monitoring unit mechanical elements (Callies et al., 2000). Although not stabilized, the detector temperatures are maintained at a sufficiently low level around $231 \mathrm{~K}$ (the actual value depends on in-orbit instrument temperature and PMD cooler settings). PMD temperatures vary somewhat and are closely linked to the optical bench temperature of the instrument. The optical bench is not temperature-stabilized and its operational temperature is between 275 and $280 \mathrm{~K}$.

In order to calculate the transmission of the atmosphere, which contains the relevant information on trace gas concentrations, the solar radiation incident on the atmosphere must be known. For this measurement a solar viewing port is located on the flight-direction side of the instrument. When this port is opened, sunlight is directed via a $\sim 40^{\circ}$ incidence mirror to a diffuser plate. Light scattered from this plate, or in general, light from other calibration sources such as the PtCrNeAr spectral light source (SLS) for wavelength calibration, and the Quartz Tungsten Halogen white light source
(WLS) for etalon (and, optionally, pixel-to-pixel gain) calibration are directed to the scan mirror using auxiliary optics. A further discussion on etalon correction is provided in Sect. 3.5. All internal calibration sources with their optics are assembled in a subsystem called the "calibration unit". The only exceptions are light-emitting diodes (LEDs) which are located in front of the detectors to monitor the pixel-to-pixel gain.

\subsection{GOME-2 band definitions and integration times}

\subsubsection{Main Focal Plane Assembly (FPA) band settings}

The GOME-2 channels can be separated into different bands. The operational band settings are provided in Table 3 . Note that the separation between band $1 \mathrm{~A}$ and band $1 \mathrm{~B}$, for GOME-2 on Metop-A, was shifted on 10 December 2008 from 307 to $283 \mathrm{~nm}$ to be consistent with the settings used for both ERS-2/GOME-1 and ENVISAT/SCIAMACHY. GOME-2 on Metop-B has also used these updated settings since the beginning of operations. Each band can be operated 
Table 3. Main channel band settings of GOME-2. The band separation change between band 1A and B occurred for GOME-2 on Metop-A during orbit 11119 on 10 December 2008. For GOME-2 on Metop-B the updated settings have been used from the beginning of operations.

\begin{tabular}{lrrrrr}
\hline Channel & Band & $\begin{array}{r}\text { Number of } \\
\text { pixels }\end{array}$ & $\begin{array}{r}\text { Approximate } \\
\text { spectral range } \\
(\mathrm{nm}) \text { Metop-A }\end{array}$ & $\begin{array}{r}\text { Approximate } \\
\text { spectral range } \\
(\mathrm{nm}) \text { Metop-B }\end{array}$ & \\
\hline 1 & $1 \mathrm{~A}$ & $877 / 659^{*}$ & $240-307 / 283^{*}$ & $239-283$ & 0.12 \\
1 & $1 \mathrm{~B}$ & $147 / 365^{*}$ & $307 / 283-314^{*}$ & $283-312$ & 0.12 \\
2 & $2 \mathrm{~A}$ & 71 & not valid & not valid & 0.12 \\
2 & 2B & 953 & $310-403$ & $308-402$ & 0.12 \\
3 & 3 & 1024 & $397-604$ & $395-604$ & 0.21 \\
4 & 4 & 1024 & $593-790$ & $593-791$ & 0.21 \\
$5 / 6$ & PMD P/S & 256 & $312-790$ & $312-790$ & $0.62-8.8$ \\
\hline
\end{tabular}

* Settings changed on 10 December 2008.

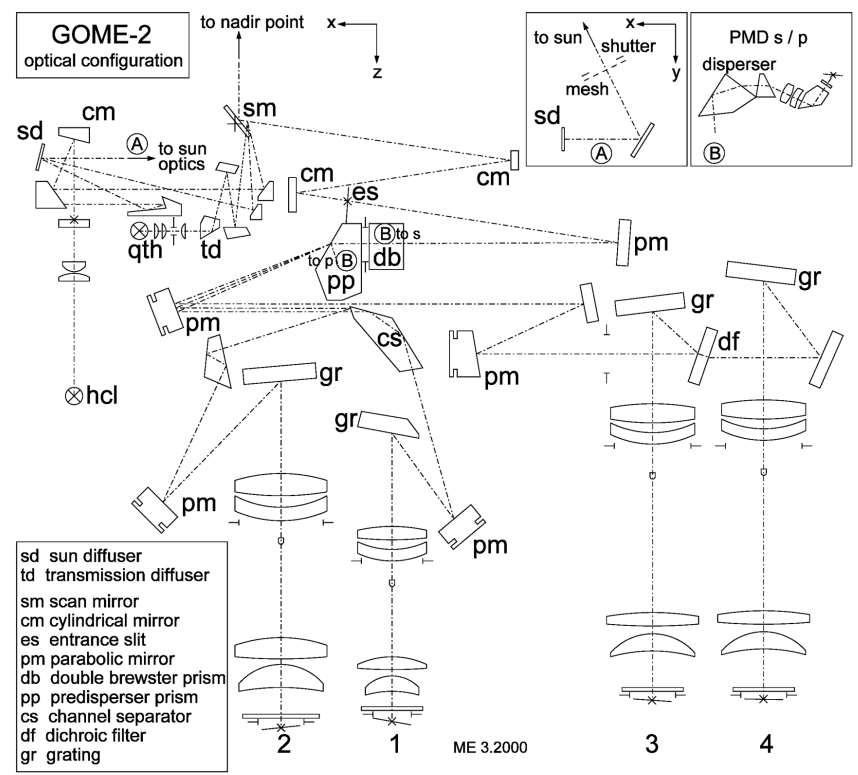

Figure 2. GOME-2 optical layout. The optics are in one plane (except insets A and B). Nadir is in $-Z$ direction (graphic provided courtesy of Selex Galileo). Note that the PtCrNeAr spectral light source (SLS) for wavelength calibration is indicated as "hcl", and the Quartz Tungsten Halogen white light source (WLS) as "qth".

at different integration times which can also vary over the orbit. The actual integration times used, and thus the ground pixel size, depend on the light intensity. Nominal integration times in band $1 \mathrm{~A}$ are $1.5 \mathrm{~s}(6 \mathrm{~s}$ at high solar zenith angles) and $0.1875 \mathrm{~s}$ for band $1 \mathrm{~B}, 2 \mathrm{~A}, 2 \mathrm{~B}, 3$, and 4 (1.5 and $0.75 \mathrm{~s}$ at high SZA). For details on the exact integration times per band during one instrument timeline series, we refer to the GOME-2 monitoring pages in the timelines subsection at http://gome.eumetsat.int.

\subsubsection{Polarization Measurement Device (PMD) band settings}

For Earth radiance measurements, the 256 used detector pixels of both PMD devices (for details we refer to EUMETSAT, 2014b) are spectrally co-added on board into 15 broader PMD spectral bands to be transmitted to ground. The PMD detector pixel numbers and spectral coverage are provided in Tables 4, 5, and 6. Before 11 March 2008, both PMD detectors (PMD-S and PMD-P) of GOME-2 on Metop-A used the same band settings, defined as a function of detector pixel, as listed in Table 4. On 11 March 2008 in orbit 7227, updated PMD band settings, with different settings for PMD-S and PMD-P were uploaded to the instrument in order to improve the spectral co-registration of both PMDs and to optimize the utility of PMD bands for level 2 data retrieval (Table 5). For GOME-2 on Metop-B the PMD band settings (Table 6) have not been changed since the beginning of operational data production.

For more details on the PMD calibration and PMD band settings we refer to EUMETSAT (2013) and EUMETSAT (2014c).

\subsection{On-ground calibration and characterization}

The GOME-2 instrument was built by an industrial team led by Selex Galileo (Italy) with support from Laben (Italy), TNO-TPD (Netherlands), Arcom Space (Denmark), Innoware (Denmark), and Finavitec (Finland) where TNOTPD is responsible for the calibration and characterization of the instrument.

Calibration and characterization measurements are taken during an extensive on-ground campaign. The detailed characterization measurements are fully described in TPD (2003) and TPD (2004a). Characterization measurements are postprocessed to provide calibration key data files which are documented both in terms of content and format in TNO (2011a, $2011 b, 2012)$. A subset of the calibration key data is a required input to the GOME-2 level 0 to 1 processor e.g. the 
Table 4. Default GOME-2 Metop-A PMD band definitions (v1.0) valid from date of launch to 11 March 2008 in orbit 7226.

\begin{tabular}{lrrrr}
\hline Band & $\begin{array}{r}\text { First pixel from } \\
\text { start of block C }\end{array}$ & Number of pixels & $\begin{array}{r}\text { Approx. wavelength of } \\
\text { start pixel (nm) }\end{array}$ & $\begin{array}{r}\text { Approx. wavelength of } \\
\text { end pixel (nm) }\end{array}$ \\
\hline 0 & 19 & 2 & 309.2 & 309.9 \\
1 & 23 & 5 & 311.7 & 314.4 \\
2 & 31 & 4 & 317.0 & 319.1 \\
3 & 37 & 12 & 321.2 & 329.5 \\
4 & 50 & 5 & 331.1 & 334.3 \\
5 & 56 & 43 & 335.9 & 377.7 \\
6 & 100 & 4 & 380.1 & 383.7 \\
7 & 115 & 20 & 399.3 & 428.4 \\
8 & 138 & 43 & 435.5 & 552.5 \\
9 & 183 & 2 & 553.6 & 557.5 \\
10 & 187 & 22 & 569.6 & 678.6 \\
11 & 217 & 2 & 742.3 & 750.2 \\
12 & 219 & 1 & 758.2 & 758.2 \\
13 & 223 & 1 & 792.1 & 792.1 \\
14 & 228 & 1 & 838.8 & 838.8 \\
\hline
\end{tabular}

Table 5. GOME-2 Metop-A PMD band definitions (v3.1). This set is valid from 11 March 2008 during orbit 7227. Here "P" indicates PMD-P which measures intensity polarized parallel to the spectrometer's slit, and "S" indicates PMD-S which measures intensity polarized perpendicular to the spectrometer's slit.

\begin{tabular}{lcrrrrrrr}
\hline Band & \multicolumn{2}{c}{$\begin{array}{c}\text { First pixel from } \\
\text { start of block C }\end{array}$} & \multicolumn{2}{c}{$\begin{array}{c}\text { Number of } \\
\text { pixels }\end{array}$} & \multicolumn{2}{c}{$\begin{array}{c}\text { Approx. wavelength of } \\
\text { start pixel (nm) }\end{array}$} & $\begin{array}{c}\text { Approx. wavelength of } \\
\text { end pixel (nm) }\end{array}$ \\
\hline & $\mathrm{S}$ & $\mathrm{P}$ & $\mathrm{S}$ & $\mathrm{P}$ & $\mathrm{S}$ & $\mathrm{P}$ & $\mathrm{S}$ & $\mathrm{P}$ \\
0 & 22 & 20 & 5 & 5 & 311.709 & 311.537 & 314.207 & 313.960 \\
1 & 30 & 29 & 4 & 4 & 316.762 & 317.068 & 318.720 & 318.983 \\
2 & 37 & 36 & 12 & 12 & 321.389 & 321.603 & 329.139 & 329.267 \\
3 & 50 & 49 & 6 & 6 & 330.622 & 330.744 & 334.443 & 334.560 \\
4 & 57 & 56 & 6 & 6 & 336.037 & 336.157 & 340.161 & 340.302 \\
5 & 84 & 83 & 17 & 17 & 360.703 & 361.054 & 377.873 & 378.204 \\
6 & 102 & 101 & 4 & 4 & 380.186 & 380.502 & 383.753 & 384.049 \\
7 & 117 & 116 & 19 & 19 & 399.581 & 399.921 & 428.585 & 429.239 \\
8 & 138 & 137 & 27 & 27 & 434.083 & 434.779 & 492.066 & 492.569 \\
9 & 165 & 164 & 18 & 18 & 494.780 & 495.272 & 548.756 & 549.237 \\
10 & 183 & 182 & 2 & 2 & 552.474 & 552.967 & 556.262 & 556.769 \\
11 & 187 & 186 & 11 & 11 & 568.070 & 568.628 & 612.869 & 613.680 \\
12 & 198 & 197 & 9 & 9 & 617.867 & 618.711 & 661.893 & 662.990 \\
13 & 218 & 217 & 4 & 4 & 744.112 & 745.379 & 768.269 & 769.553 \\
14 & 224 & 223 & 2 & 2 & 794.080 & 795.364 & 803.072 & 804.351 \\
\hline
\end{tabular}

radiance, irradiance, and polarization response of the instrument.

For a full list of those key data used by the GOME-2 level 0 to $1 \mathrm{~b}$ processing chain see EUMETSAT, 2014b. Other key data describe aspects of the on-ground behaviour of the instrument which will also be measured in-orbit using onboard calibration targets e.g. dark-signal performance, pixelto-pixel gain, spectral calibration, and etalon.

The slit-function shape must also be characterized at subpixel resolution pre-flight, as this cannot be determined adequately from information available in-flight. As a result further slit-function characterization data, additional to that available from the baseline calibration and characterization activities, were also acquired during the on-ground calibration and characterization campaign (TPD, 2004b). These data were further analysed by TNO-TPD and the Rutherford Appleton Laboratory to provide calibration key data that describe the slit-function shape at sub-pixel resolution for use in level 1 to 2 processing. Calibration key data sets, related documents, and slit-function characterization data are available from the GOME-2 monitoring pages in the documentation subsection at http://gome.eumetsat.int. 
Table 6. GOME-2 Metop-B PMD band definitions (v2.0). This set is valid from 29 October 2012 during orbit 598. Here "P" indicates PMD-P which measures intensity polarized parallel to the spectrometer's slit, and " $\mathrm{S}$ " indicates PMD-S which measures intensity polarized perpendicular to the spectrometer's slit.

\begin{tabular}{lcrrrrrrr}
\hline Band & \multicolumn{2}{c}{$\begin{array}{c}\text { First pixel from } \\
\text { start of block C }\end{array}$} & \multicolumn{2}{c}{$\begin{array}{c}\text { Number of } \\
\text { pixels }\end{array}$} & \multicolumn{2}{c}{$\begin{array}{c}\text { Approx. wavelength of } \\
\text { start pixel }(\mathrm{nm})\end{array}$} & \multicolumn{2}{c}{$\begin{array}{c}\text { Approx. wavelength of } \\
\text { end pixel (nm) }\end{array}$} \\
\hline & $\mathrm{S}$ & $\mathrm{P}$ & $\mathrm{S}$ & $\mathrm{P}$ & $\mathrm{S}$ & $\mathrm{P}$ & $\mathrm{S}$ & $\mathrm{P}$ \\
0 & 22 & 23 & 5 & 5 & 311.875 & 312.001 & 314.375 & 314.516 \\
1 & 30 & 31 & 4 & 4 & 316.948 & 317.102 & 318.926 & 319.090 \\
2 & 37 & 38 & 12 & 12 & 321.634 & 321.807 & 329.514 & 329.675 \\
3 & 50 & 51 & 6 & 6 & 331.016 & 331.172 & 334.872 & 335.020 \\
4 & 56 & 57 & 6 & 6 & 335.661 & 335.808 & 339.698 & 339.848 \\
5 & 84 & 85 & 17 & 17 & 360.644 & 360.825 & 378.138 & 378.308 \\
6 & 102 & 103 & 4 & 4 & 380.515 & 380.706 & 384.170 & 384.399 \\
7 & 117 & 118 & 19 & 19 & 400.059 & 400.383 & 428.350 & 428.763 \\
8 & 139 & 140 & 27 & 27 & 435.521 & 435.971 & 493.181 & 493.963 \\
9 & 166 & 167 & 18 & 18 & 495.886 & 496.683 & 549.510 & 550.625 \\
10 & 184 & 185 & 2 & 2 & 553.192 & 554.330 & 556.944 & 558.106 \\
11 & 188 & 189 & 11 & 11 & 568.637 & 569.885 & 612.854 & 614.699 \\
12 & 199 & 200 & 8 & 8 & 617.781 & 619.714 & 656.047 & 658.343 \\
13 & 218 & 219 & 4 & 4 & 737.179 & 739.726 & 760.682 & 763.366 \\
14 & 224 & 225 & 2 & 2 & 785.719 & 788.551 & 794.415 & 797.300 \\
\hline
\end{tabular}

\subsection{In-flight characterization and calibration}

A range of in-flight characterization and calibration activities are carried out routinely during GOME- 2 operations. These activities provide input to level 0 to $1 \mathrm{~b}$ processing, in addition to the calibration key data measured on-ground, to ensure the generation of high-quality spectrally and radiometrically calibrated radiance and irradiance data and continuous monitoring of instrument performance.

Calibration activities interleaved with nominal observations comprise dark-signal measurements performed every orbit in eclipse, and sun calibration measurements performed once per day at sunrise in the Northern Hemisphere. The sun calibration uses one of the on-board stored timelines which includes in addition to the sun measurements itself, both a wavelength calibration and a radiometric calibration. The timeline must be triggered such that the instrument is commanded into solar measurement observation mode prior to the sun appearing in the instrument field of view. For the remainder of the orbit the timeline consists of nadir scanning observations.

In addition, regular monthly calibration activities take place. The frequency is determined by the expected change in the mean optical bench temperature, resulting from seasonal variations in the external heat load from the sun, and longterm degradation of thermo-optical surfaces. Although it is primarily the wavelength calibration that is expected to vary, diffuser characterization and other calibration and monitoring activities are carried out during the monthly calibration activities.

\subsection{GOME-2 observation modes}

\subsubsection{Earth observation modes}

Earth observation (or "Earth radiance") modes are those modes where the Earth is in the field of view of GOME-2. They are usually employed on the dayside of the Earth (sunlit part of the orbit). The scan mirror can be at a fixed position (static modes), or continuously scan around a certain position (scanning modes). All internal light sources are switched off and the solar port of the calibration unit is closed.

- Nadir scanning: this is the mode in which GOME-2 is operated most of the time. The scan mirror performs a nadir swath as described above.

- Nadir static: the scan mirror points towards nadir. This mode is typically used during the monthly calibration. It is valuable for validation and long-loop sensor performance monitoring purposes.

\subsubsection{Calibration modes}

In-orbit instrument calibration and characterization data are acquired in the various calibration modes. They are usually employed during eclipse, with the exception of the solar calibration which is performed at sunrise. Both internal (WLS, SLS, LED) and external (sun, moon) light sources can be employed. The various sources are selected by the scan mirror position.

- Dark: the scan mirror points towards the GOME-2 telescope. All internal light sources are switched off and the 
solar port is closed. Dark signals are typically measured every orbit during eclipse.

- Sun (over diffuser): the scan mirror points towards the diffuser. All internal light sources are switched off and the solar port is open. Solar spectra are typically acquired once per day at the terminator in the Northern Hemisphere. The sun mean reference (SMR) spectra are derived from this mode.

- White light source (direct): the scan mirror points towards the WLS output mirror. The WLS is switched on and the solar port is closed. Etalon (and optionally pixel-to-pixel gain (PPG) calibration) data are derived from this mode.

- Spectral light source (direct): the scan mirror points towards the SLS output mirror. The SLS is switched on and the solar port is closed. Wavelength calibration coefficients are derived from this mode.

- Spectral light source over diffuser: the scan mirror points towards the diffuser. The SLS is switched on and the solar port is closed. Light from the SLS reaches the scan mirror via the diffuser. This mode is intended to be employed for in-orbit monitoring of the sun diffuser reflectivity. In practise, however, this instrument mode has not been particularly useful as the SLS has proven to be unstable for the long integration times required to measure over the sun diffuser. Analysis of these measurements has only been used to provide a rough estimate of the upper boundary for diffuser degradation.

- LED: the scan mirror points towards the GOME-2 telescope. The LEDs are switched on and the solar port is closed. Pixel-to-pixel gain (PPG) calibration data are derived from this mode.

- Moon: the scan mirror points towards the moon (typical viewing angles are $+70^{\circ}$ to $+85^{\circ}$ ). As the spacecraft moves along the orbit, the moon passes the GOME-2 slit within a few minutes. This mode can be employed only if geometrical conditions (lunar azimuth, elevation, and pass angle) allow, which typically occur a few times per year.

\subsection{GOME-2 instrument operations plan}

The default swath width of the scan is $1920 \mathrm{~km}$ which enables global coverage of the Earth's surface within 1.5 days. The scan mirror speed can be adjusted such that, despite the projection effect, the ground is scanned across-track at constant speed. The along-track dimension of the instantaneous field of view (IFOV) is $\sim 40 \mathrm{~km}$ which is matched with the spacecraft velocity such that each scan closely follows the ground coverage of the previous one. The IFOV acrosstrack dimension is $\sim 4 \mathrm{~km}$. For the $1920 \mathrm{~km}$ swath, the max- imum temporal resolution of $187.5 \mathrm{~ms}$ for the main channels (23.4 ms for the PMD channels) corresponds to a maximum ground pixel resolution (across track $\times$ along track) of $80 \mathrm{~km} \times 40 \mathrm{~km}(10 \mathrm{~km} \times 40 \mathrm{~km}$ for the PMDs $)$ in the forward scan. See also Sect. 1.7 for more information on the scan and data packet structure. Since 15 July 2013, both GOME-2 instruments on board Metop-A and Metop-B have been operated in tandem with the swath width of GOME-2 on Metop-A changed to $960 \mathrm{~km}$, while GOME-2 on Metop$B$ remains with the full swath width of $1920 \mathrm{~km}$. This results in a ground pixel size (across track $\times$ along track) of $40 \times 40 \mathrm{~km}$ for GOME-2 on Metop-A main science channels $(5 \times 40 \mathrm{~km}$ for the PMDs) and $80 \times 40 \mathrm{~km}$ for GOME- 2 on Metop-B main science channels $(10 \times 40 \mathrm{~km}$ for the PMDs $)$.

The 29-day instrument operations plans for Metop-A and Metop-B are shown in Figs. 3 and 4. The monthly calibration sequence is activated for both instruments on the same day with two nadir static orbits (no scanning) followed by one orbit during which the PMD data are downlinked at full spectral resolution (256 channels for both PMDs) but with a reduced number of read-outs (12 read-outs in forward and 4 read-outs in backward scanning direction whilst retaining nominal spatial resolution for PMDs, i.e. with gaps in between the read-outs). This configuration is called the monthly PMD RAW read-out configuration. GOME-2 on Metop-A is also configured to operate once per month with a reduced swath width of $320 \mathrm{~km}$. For further information see the GOME-2 monitoring pages in the timelines subsection at $\mathrm{http} / / /$ gome.eumetsat.int. For more details on GOME-2 operations and the instrument settings, see EUMETSAT (2014a).

\subsection{GOME-2 data packet structure and synchronization of measurements}

A basic concept in the operation of the GOME-2 instrument is that of the "scan". A scan is defined as a time interval of $6 \mathrm{~s}$, consisting of 16 subsets of $375 \mathrm{~ms}$ each, equivalent to one data packet. The subsets are numbered from 0 to 15 . In the Earth scanning mode, a scan consists of one scan cycle: $4.5 \mathrm{~s}$ forward scan (subsets 0 to 11) and $1.5 \mathrm{~s}$ fly-back (subsets 12 to 15$)$. In the static and calibration modes the scan mirror does not move, but the data packet structure is identical to the scanning mode.

In the default measuring mode, the nadir scan, the scan mirror sweeps in $4.5 \mathrm{~s}$ (12 subsets) from negative (east) to positive (west) viewing angles, followed by a fly-back of $1.5 \mathrm{~s}$ (the last four subsets) back to negative viewing angles as shown in Fig. 5.

GOME-2 creates a science data packet every $375 \mathrm{~ms}$. One packet contains one UTC time stamp $t_{0}$, four scanner positions (sampling $93.75 \mathrm{~ms}$ ), at most two read-outs of the main channels (effective integration time $187.5 \mathrm{~ms}$ ), and at most 16 read-outs of the PMD bands (integration time $23.4375 \mathrm{~ms}$ ).

The relative timing of the read-outs is shown in Fig. 6. The first scanner position is given at time $t_{0}+\Delta t_{\mathrm{SM}}$ (the time delta 


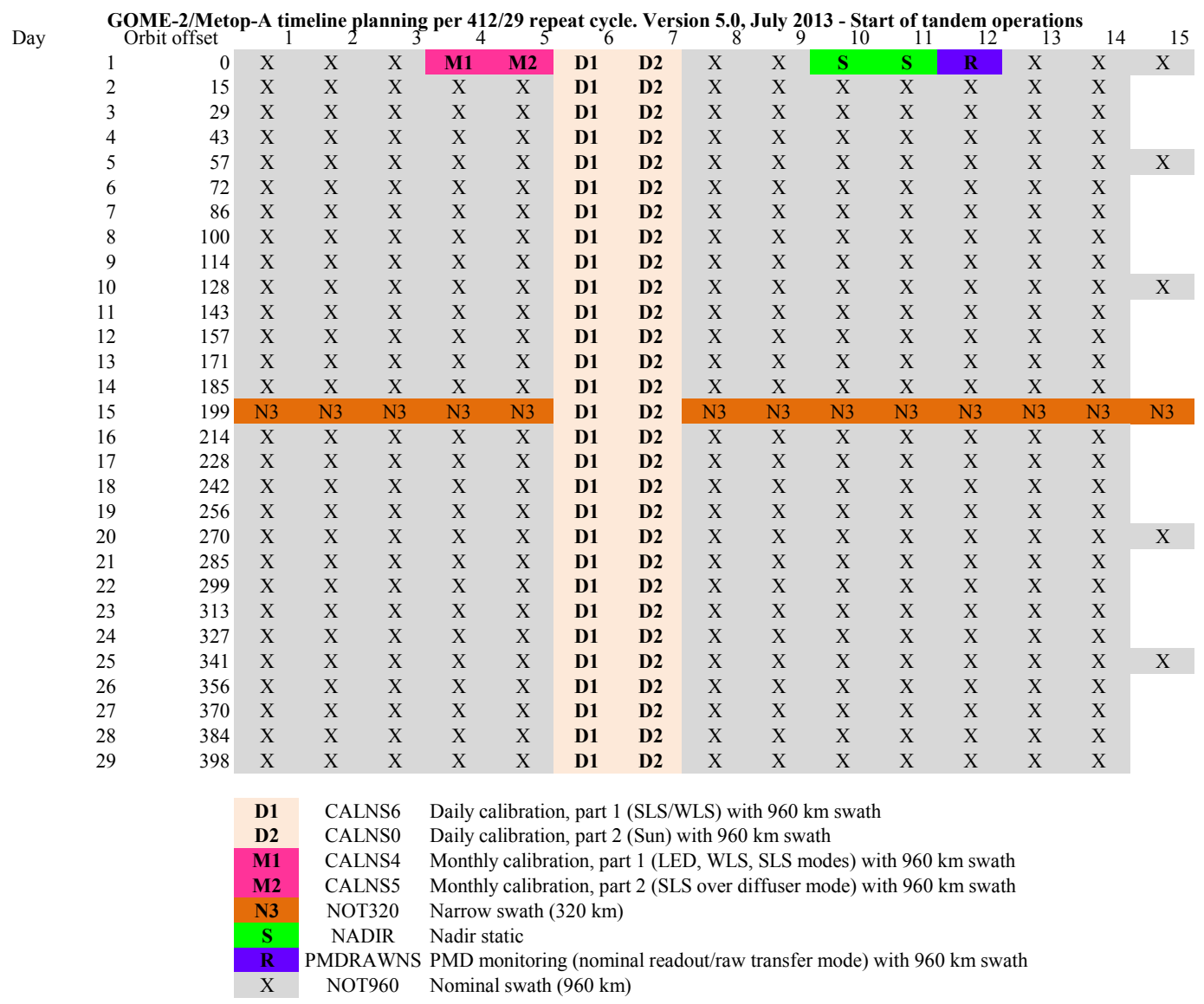

Figure 3. The 29-day instrument operation cycle for GOME-2 on Metop-A. Different colours and ID tags indicate different instrument operations settings as explained in the legend.

for the scan mirror between one data packet and the next). At the same time, the first main channel read-out and the first PMD read-out within the packet start. The read-out time is $45.78 \mu$ s per detector pixel, i.e. $46.875 \mathrm{~ms}$ for a complete main channel read-out (1024 detector pixels), and $11.72 \mathrm{~ms}$ for a read-out of PMD blocks B,C, and D (256 detector pixels, see EUMETSAT, 2014a). Any read-out operation resets a detector pixel, and therefore marks the end of the integration for this detector pixel. The integration starts at the previous read-out.

The read-out sequence for each detector can be programmed to be either from short to long wavelengths ("up") or from long to short wavelengths ("down").

\section{GOME-2 Level 0 to 1 data processing}

GOME-2 data level 0 data are processed centrally at EUMETSAT in near-real time and made available to users through a variety of mechanisms. More information on data access can be found at www.eumetsat.int $>$ Data $>$ Data De- livery. The data processing is separated into two main stages: level 0 to $1 \mathrm{a}$ processing and level $1 \mathrm{a}$ to $1 \mathrm{~b}$ processing.

\subsection{Level 0 to 1a processing}

The level 0 to $1 \mathrm{a}$ processing comprises both the determination of geolocation information on a fixed time grid, and the determination of applicable calibration parameters. A schematic overview of the level 0 to 1a processing steps and their applicability for different observation modes is available for reference in EUMETSAT (2014b), Figs. 5, 6, 7, and 8 . From measurements of the various calibration sources encountered during each run of the processor, new calibration constants are calculated and written into an in-flight calibration data file. They are also retained in memory for use in processing those data acquired after the satellite comes out of the dark side of the orbit and before the next dump. Updated calibration parameters are available for immediate use. Calibration parameters are stored for the lifetime of the mission. The calibration constant determination comprises the following:

- dark current correction 


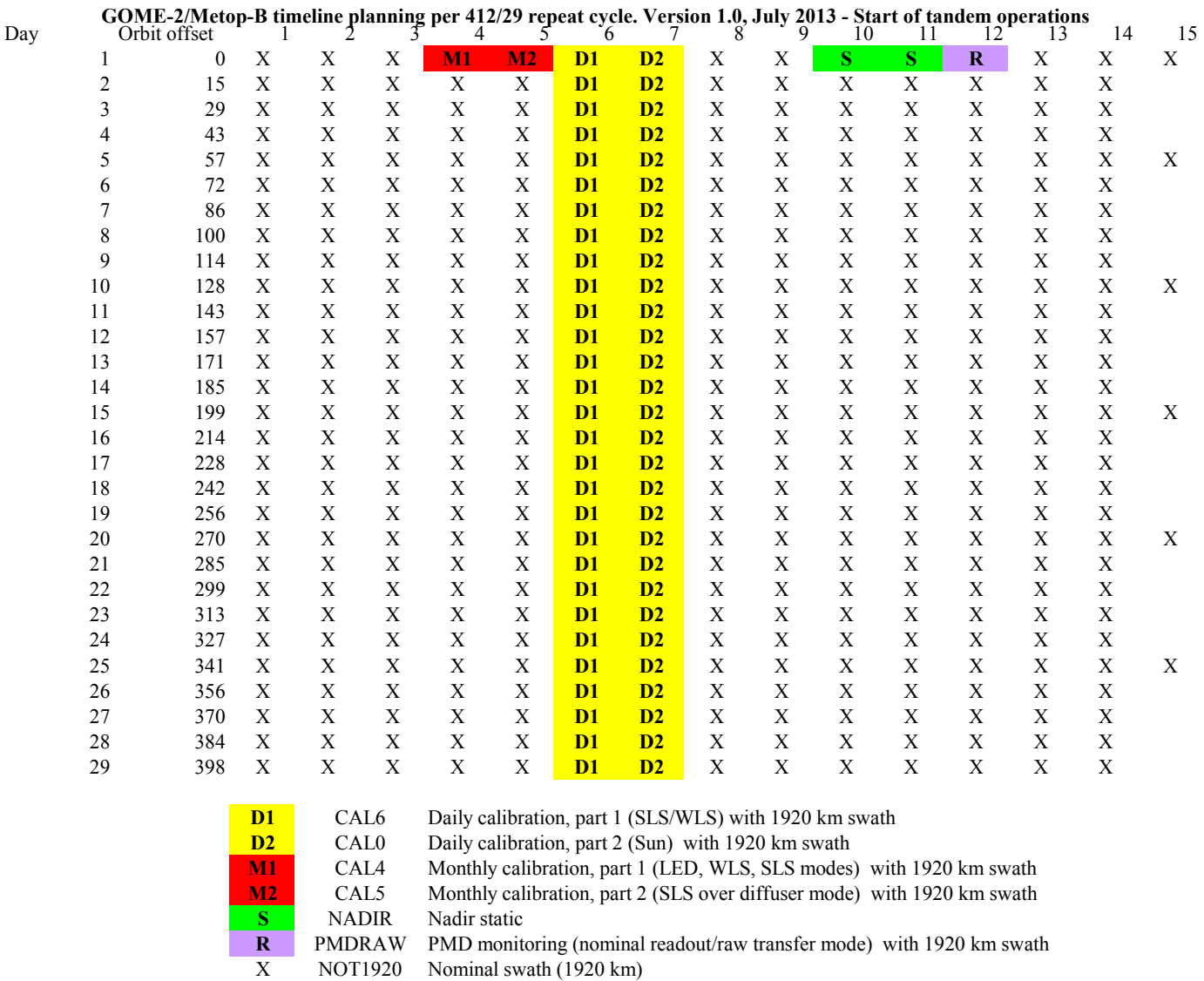

Figure 4. The 29-day instrument operation cycle of GOME-2 on Metop-B. Different colours and ID tags indicate different instrument operations settings as explained in the legend.
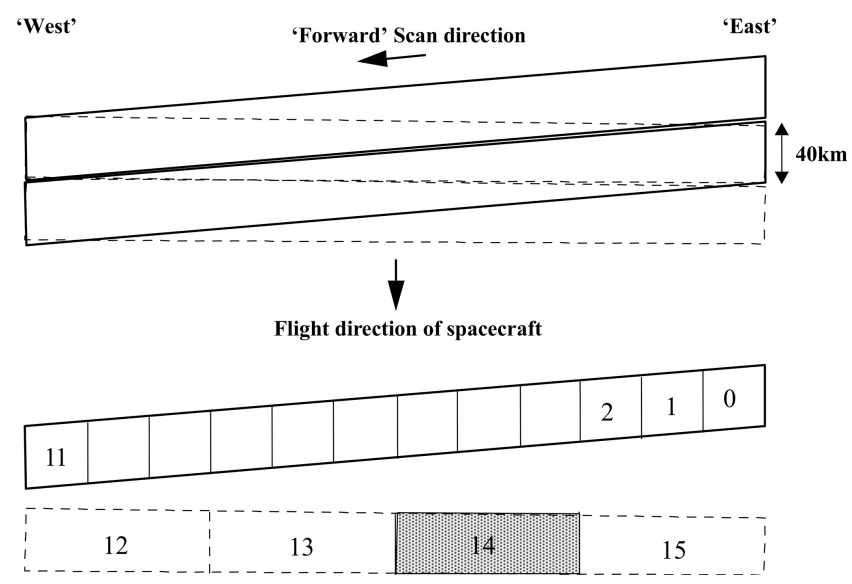

Figure 5. Scan pattern in default scan mode. Solid line: forward scan; dashed line: fly back. Each subset pixel (0-15) corresponds to $375 \mathrm{~ms}$. In one of the four subsets of the fly back (subset 14 is shown as an example only) the "unused" parts of the PMD detectors (i.e. block A; see Appendix B of EUMETSAT, 2014a) are read out.
- pixel-to-pixel gain correction

- determination of spectral calibration parameters

- etalon correction

- determination of stray light correction factors for the sun and polarization measurements

- determination of the solar mean reference spectrum, and atmospheric polarization state.

The geolocation of the measurements is calculated from the appropriate orbit and attitude information, and time correlation information in the level 0 data stream. Note, any application of calibration parameters in the level 0 to $1 \mathrm{a}$ processing should be regarded as interim, to facilitate the generation of new calibration parameters and correction factors. There is no application of calibration parameters to main channel Earth observation measurements. The output of the level 0 to $1 \mathrm{a}$ processor is formatted into the level 0 and 1a products as specified in EUMETSAT (2010, 2014d). 


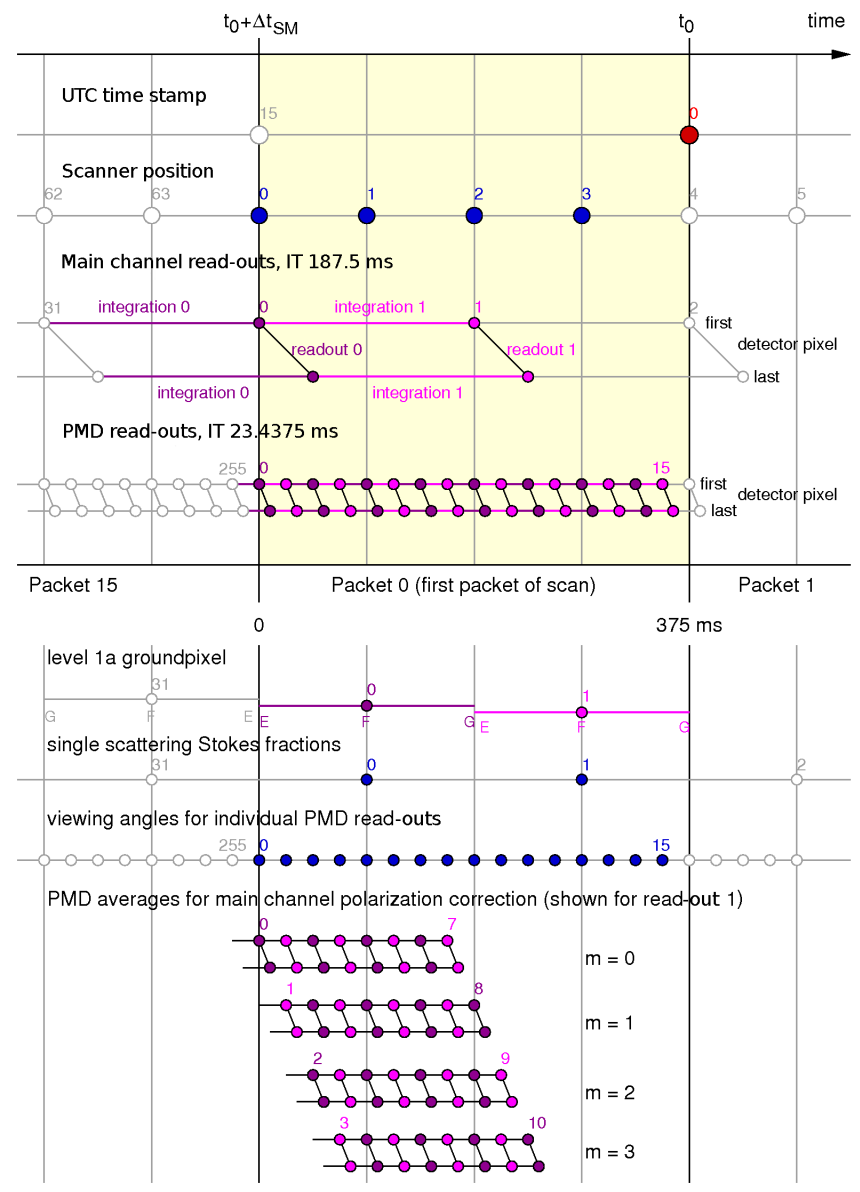

Figure 6. Synchronization diagram. Synchronization of time stamp, scanner positions, and detector read-outs within a GOME-2 science data packet, and selected quantities of the level 0 to 1a processing. The data transmitted in packet 0 (the first of a scan) are indicated by the filled circles. Open circles belong to the previous or next data packet. For this figure, $\Delta t_{\mathrm{SM}}$ was assumed to be $-375 \mathrm{~ms}$. The indexing refers to a complete scan.

\subsection{Level 1a to $1 \mathrm{~b}$ processing}

The level 0 to $1 \mathrm{~b}$ processing comprises the calculation of geolocation parameters for the actual integration time of each measurement, determination of stray light correction factors for the Earth radiance measurements, and the conversion of the raw binary read-outs on the level 1a data stream to calibrated radiance and irradiance data. Cloud parameters are also determined. Furthermore, calibrated measurements from the on-board calibration sources, and the sun and moon are available in the level $1 \mathrm{~b}$ product. Cloud parameters are also determined and added to the level $1 \mathrm{~b}$ product. A description of the algorithms used to derive the cloud parameters can be found in Sect. 3.9. A schematic overview of the level 1a to $1 \mathrm{~b}$ processing steps and their applicability for different observations modes is available for reference in EUMET-
SAT (2014b), Figs. 11, 12, 13, and 14. Level 1b data are formatted as specified in EUMETSAT (2010, 2014d).

\section{GOME-2 Level 0 to 1 algorithms}

\subsection{Geolocation and synchronization of main channel and PMD data}

Basic geolocation parameters such as the sub-satellite point and solar angles at the satellite are calculated for all measurement modes. In addition, mode-specific geolocation parameters are calculated for Earth, sun, and moon modes as follows:

- Earth mode: solar and line-of-sight zenith and azimuth angles at a given height, corner and centre coordinates (latitude/longitude) of the ground pixel at ground level, satellite height, and Earth radius.

- sun mode: distance between satellite and sun and relative speed of satellite and sun.

- moon mode: Lunar elevation and azimuth angles, sunmoon distance, satellite-moon distance, lunar phase angle, illuminated fraction of lunar disk.

Geolocation parameters are processed during level 0 to $1 \mathrm{~b}$ processing both for a fixed grid of 32 read-outs per scan and for an individual grid based on the actual integration time of an instrument band.

Figure 7 shows the orientation of the GOME-2 ground pixel within the scan and relative to the flight direction. Letters A-F indicate the position, relative to the ground pixel, of points for which geolocation information is provided in the GOME-2 level $1 \mathrm{~b}$ product.

Note that geolocation information is provided in the GOME-2 level $1 \mathrm{~b}$ product only for the first detector pixel of each channel (EUMETSAT, 2010, 2014d). Therefore for a proper assignment of geolocation to the GOME-2 measurements and for a correct combination of main channel and PMD data, the synchronization of UTC time stamps, scan mirror positions and detector read-outs in the science data packet have to be understood, and the relative timing of the main channel and PMD read-outs, and the finite duration of the detector read-outs, must be accounted for.

Specifically, the user of the geolocation information needs to be aware that the so-called effect of "spatial aliasing" has to be accounted for when very accurate geo-referencing or co-registration of main channel and PMD data is needed. The spatial aliasing effect arises because the individual detector pixels are read out sequentially whilst the scan mirror is still moving. As a result every detector pixel of each detector sees a slightly different ground scene and thus spatial information is "aliased" into spectral information. The detector arrays have been programmed to read out in alternating direction so that the last and first detector pixels of spectrally adja- 

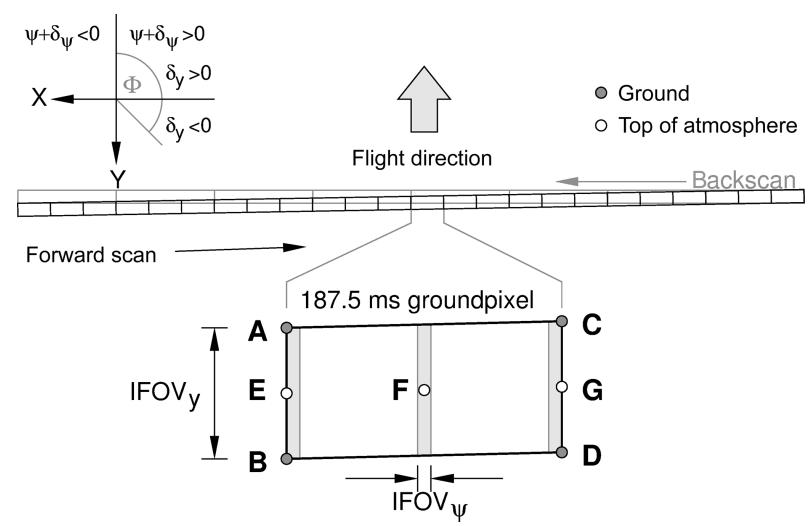

Figure 7. GOME-2 ground pixel orientation relative to the scan and the flight direction.

cent channels see approximately the same scene, thus minimizing discontinuities between channels. The read-out direction is specified in the field CHANNEL_READOUT_SEQ of GIADR-Channels record in the GOME-2 level $1 \mathrm{~b}$ product (EUMETSAT, 2010, 2014d).

Knowing the time taken to read out a single detector pixel $\delta_{\mathrm{rd}}=45.78 \mu \mathrm{s}$ it is possible to calculate the time taken from the read-out of the first detector pixel, for which geolocation information is provided, to the read-out of the detector pixel of interest and thereby calculate accurate timing and geolocation information for the pixel of interest.

For detector pixel $i$ of channel $j$ of the main science channels, the time delay is calculated as

$t_{i j, \mathrm{FPA}}=\left\{\begin{array}{l}i \times \delta_{\mathrm{rd}}(\mathrm{read}-\text { out sequence "up" }) \\ (1023-i) \times \delta_{\mathrm{rd}}(\mathrm{read}-\text { out sequence } " \text { down" })\end{array}\right.$

and for detector pixel $k$ of the two polarization detectors, the time delay is calculated as

$t_{k, \mathrm{PMD}}=\left\{\begin{array}{l}(k-768) \times \delta_{\text {rd }}(\mathrm{read}-\text { out sequence "up" }) \\ (1023-k) \times \delta_{\text {rd }}(\text { read }- \text { out sequence "down" }) .\end{array}\right.$

To calculate the shift in geolocation parameters resulting from this time delay related to detector read-out, a simple linear interpolation between the geolocation parameters provided for the first detector pixel of read-out $n$ and read-out $n+1$ is sufficient.

Therefore, for main science channel data,

$$
\begin{aligned}
& \text { geolocation }_{i, \text { actual }}=\frac{t_{i j, \mathrm{FPA}}}{\mathrm{IT}_{\mathrm{FPA}}} \\
& \quad \times\left(\text { geolocation }_{n+1}-\text { geolocation }_{n}\right)+\text { geolocation }_{n}
\end{aligned}
$$

and for polarization channel data,

$$
\begin{aligned}
& \text { geolocation }_{k, \text { actual }}=\frac{t_{k, \mathrm{PMD}}}{\mathrm{IT}_{\mathrm{PMD}}} \\
& \times\left(\text { geolocation }_{n+1}-\text { geolocation }_{n}\right)+\text { geolocation }_{n},
\end{aligned}
$$

where $\mathrm{IT}_{\mathrm{PPA}}$ and $\mathrm{IT}_{\mathrm{PMD}}$ are the integration times in use for the main channels and PMDs respectively, which determine

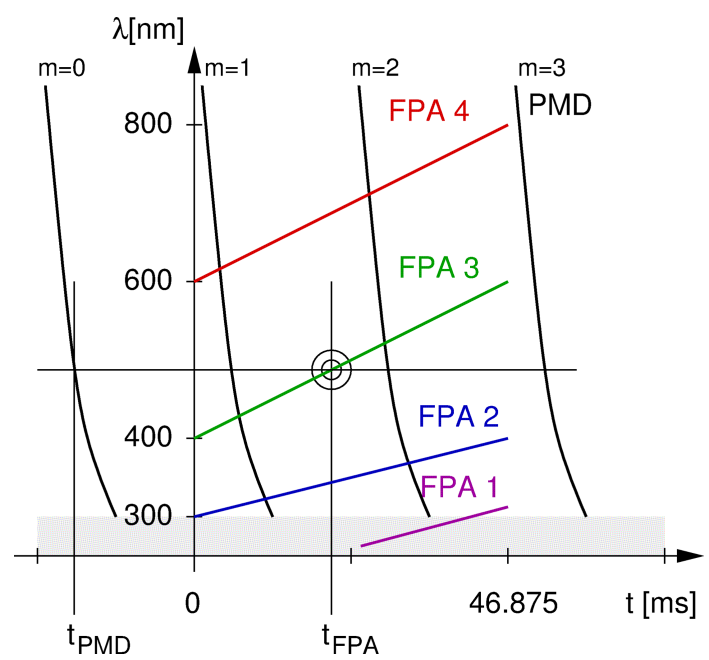

Figure 8. Timing diagram for main channel and PMD detector pixel read-outs. For this example, read-out sequence "up" has been assumed for the four main channels, and read-out sequence "down" for the two PMD channels. Readout of the main channels and readout of the PMD channels for $m=1$ starts at $t=0$. For a given detector pixel in a main channel, indicated by the circles, its read-out time $t_{\mathrm{FPA}}$ and the one of the PMD pixel closest in wavelength, $t_{\mathrm{PMD}}$ are determined. The difference between the two times is then used for linear interpolation of the PMD data between the two adjacent PMD curves.

the time difference between successive read-outs for which geolocation information of the first detector pixel is available.

In case the user wishes to co-register main channel and PMD data care must also be taken to account for the synchronization between the measurements as shown in Fig. 6 so that the main channel and PMD data correspond as closely as possible to the same ground scene. This is needed for example when PMD data are co-registered with main channel data for the derivation of the polarization correction, or when coregistering cloud information provided at PMD resolution (see Sect. 3.9) with main channel data. Here the relative timing between the read-out of a main channel detector pixel and the read-out of a PMD pixel at the same wavelength must be considered. This is determined by the read-out time per detector pixel, the read-out sequence of the GOME-2 channels ("up" or "down"), and their dispersion relation (pixel - wavelength correspondence); see Fig. 8.

First it is necessary to create four sets of eight averaged PMD read-outs $\left(\bar{p}_{m}\right.$ where $\left.m=0 \cdots 3\right)$ for each main $187.5 \mathrm{~ms}$ channel read-out, each shifted by one PMD readout $(23.4 \mathrm{~ms})$ with respect to each previous set. See Fig. 6 for a graphical representation in the case of use of PMD data for polarization correction and also Fig. 8 .

Using the PMD wavelength grid, the PMD detector pixel $k$ which is closest in wavelength to the main channel wavelength $\lambda_{i j}$ for detector pixel $i$ of channel $j$ of the main sci- 


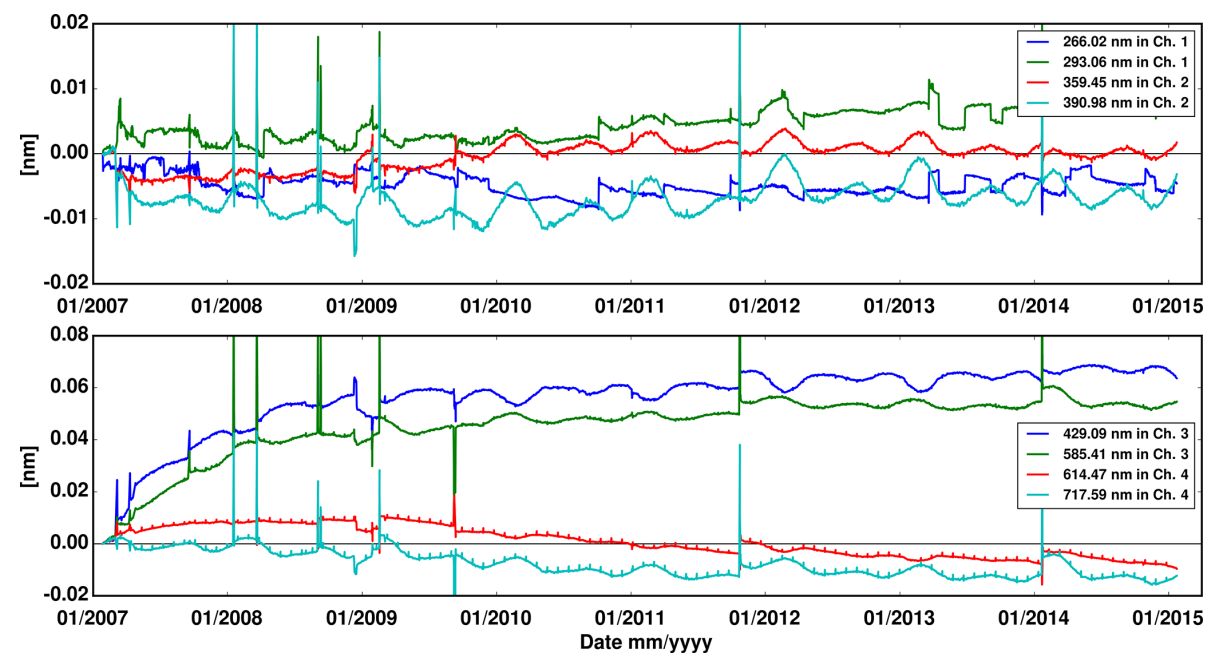

Figure 9. Difference of the centre line position with respect to January 2007 evaluated from a regular Gaussian fitting of well-separated SLS lines. The upper panel shows the results in channel 1 and 2 (blue line $266.02 \mathrm{~nm}$ in channel 1, green line $293.06 \mathrm{~nm}$ in channel 1 , red line $359.45 \mathrm{~nm}$ in channel 2, and turquoise line $390.98 \mathrm{~nm}$ in channel 2). The lower panel shows results for channel 3 and 4 (blue line $429.09 \mathrm{~nm}$ in channel 3, green line $585.41 \mathrm{~nm}$ in channel 3, red line $614.47 \mathrm{~nm}$ in channel 4, and turquoise line $717.59 \mathrm{~nm}$ in channel 4).

ence channels, is determined. Each main channel detector pixel is then assigned a parameter $m^{r}$ expressing its timing relative to the timing of the PMD data for $m=0$ calculated as:

$$
\begin{aligned}
m_{i j}^{r}= & \left(t_{i j}, \mathrm{FPA}-t_{k}, \mathrm{PMD}\right) /\left(t_{i j}, \mathrm{FPA}-t_{k}, \mathrm{PMD}\right) \\
& 23.4375 \mathrm{~ms}+123.4375 \mathrm{~ms}+1,
\end{aligned}
$$

where $23.4375 \mathrm{~ms}$ is the integration time of a single PMD measurement. The start of the main channel read-out is chosen as the time origin $(t=0)$. This corresponds to the start of the last read-out of the PMD channels which have been averaged into set $m=1$ (see Fig. 8). For example, if a pixel has $m^{r}=1.6$ (highlighted in Fig. 8) it would correspond to a measurement $0.6 \cdot 23.4 \mathrm{~ms}$ later than the PMD data from set $m=1$, and $0.4 \cdot 23.4 \mathrm{~ms}$ before the PMD data from set $m=2$.

If $m^{r}$ is split into its integer part $m^{r \text {,int }}$ and its fractional part $m^{r \text {,frac }}$ using a modulus function, the four sets $\left(\bar{p}_{m}\right.$ where $\left.m=0 \cdots 3\right)$ of averaged PMD information can be reduced to a single set $p_{\mathrm{s}}$, taking into account the relative timing between main channels and PMD channels, by calculating

$p_{\mathrm{s}, i j}=\left(1-m_{i j}^{r \text {,rac }}\right) \cdot \bar{p}_{i j, m}+m_{i j}^{r \text { frac }} \cdot \bar{p}_{i j, m+1}$

where $m=m_{i j}^{r \text {,int }}$.

\subsection{Dark-signal correction}

The dark signal for each combination of integration time and detector temperature encountered in nominal operations is calculated as an average of measurements taken in dark mode during eclipse. Applying the dark-signal correction means subtracting the appropriate dark signal, for a given combination of integration time and detector temperature, from a spectrum. The dark-signal correction is applied only for the relevant integration time and within a narrow range of the actual temperature. This is the most basic and the first of all corrections applied in level 0 to 1 processing.

The dark signal has two components: the integration-timeindependent offset of typically 1500 binary unit (BU) which is equivalent to $\sim 10^{12}$ photons $/ \mathrm{cm}^{2} \cdot \mathrm{nm} \cdot \mathrm{sr}$ at $500 \mathrm{~nm}$ (for comparison the signal in the visible region typically lies in the range 3000 to $30000 \mathrm{BU}$ depending on integration time, wavelength, viewing geometry, and observed scene), and the integration-time and detector-temperature-dependent leakage current of typically $0.7 \mathrm{BU} \mathrm{s}^{-1}$ at a detector temperature of $235 \mathrm{~K}$. When calculating and applying the dark-signal correction, only the total dark signal is considered. There is no need to split the dark signal into its components. This is only done for long-term monitoring purposes. The status of the dark-signal performance is regularly reviewed by the annual GOME-2 instrument review (EUMETSAT, 2015a, b).

The baseline for the electronic offset is steadily increasing for all bands. However, the increase is very small (single BU level). The leakage current is increasing moderately and at a level of less than $0.5 \mathrm{BU} \mathrm{s}^{-1}$ per year, which is not unexpected for this detector type and was also observed for GOME/ERS-2 which used detectors of the same type (Coldewey-Egbers, 2008).

In addition the noise is very stable across the spectrum and slightly below $2 \mathrm{BU}$, as expected from pre-flight calibrations. There is no negative impact from the very small increase in dark-signal electronic offset on the product quality, or the signal-to-noise ratio. 


\subsection{Pixel-to-pixel gain (PPG) correction}

Given a uniform illumination over the detector array, darksignal-corrected signals vary slightly between detector pixels, mainly because of small differences in pixel width. To compensate for this effect, the variation in pixel-to pixel gain is determined from measurements in LED mode which provide a fairly uniform illumination in the green part of the spectrum $(\sim 570 \mathrm{~nm})$. PPG is not expected to be dependent on the wavelength of the incident light. A PPG correction spectrum is obtained by fitting a third-order polynomial smoothing function to the LED (fall back: WLS) measurements and dividing the measured spectrum by the smoothed spectrum. The PPG correction is of the order of $10^{-4}$ relative with some increase in the PPG correction for channel 1 and 2 towards the end of the time period for Metop-A. The contribution of this signal to the overall throughput degradation and variation of calibrated radiances is however small.

\subsection{Spectral calibration and instrument spectral response function}

Spectral calibration is the assignment of a wavelength value to each detector pixel. For each GOME-2 channel, a loworder polynomial approximation is used to describe wavelength as a function of detector pixel. Polynomial coefficients are derived from preprocessed spectra of the on-board SLS which provides a number of spectral lines at known wavelengths across the GOME-2 wavelength range. Different algorithms are used for FPA and PMD channels because their spectral resolution is different. Individual spectral lines can only be resolved in the FPA channels. Positions of individual lines from a predefined set are determined using a Falk centre-of-gravity algorithm. For the PMD channels an iterative cross-correlation algorithm is used. The expected PMD signal is calculated from the measured FPA signal, taking into account the PMD slit function and ratio of radiometric response between PMD and FPA channels. The expected PMD signal is then spectrally matched with the measured PMD signal using cross correlation.

The detector pixel onto which light of a given wavelength is impinging depends on instrument temperature. The temperature at the pre-disperser prism is used as a reference. Typical shifts with temperature are of the order of 0.01 detector pixel/K for the FPA channels, but depend on the detector pixel.

Currently one spectral calibration is carried out on board every day. Spectral stability in orbit, which is a function of pre-disperser prism temperature, is very good. However, there is a very well-known orbital variation in spectral assignment $(<0.01 \mathrm{~nm})$ due to the orbital change of the optical bench temperature which is not thermally stabilized. Further adjustments to the spectral calibration can be made using Fraunhofer lines in the solar and/or Earth measurements (van Geffen, 2004) or during level 2 product production us- ing shift and squeeze fitting techniques (Beirle et al., 2013). Also, there are some changes on short timescales due to instrument switch-off events. Overall the spectral calibration stability is well within the spectral range of a detector pixel. Figure 9 shows the difference in the centre line positions with respect to November 2007. The change in the centre line position does not exhibit any long-term trend (with the exception of a small drift in channel 3 prior to 2009) and is primarily correlated with seasonal changes (and orbital as known from level 2 retrievals) of the optical bench temperature.

The spectral stability of PMD-P with respect to PMD-S is also an important quantity since the stability of the spectral co-registration of the two polarization detector wavelength grids affects the quality of the derived Stokes fraction quantities. This is in turn key to the accurate polarization correction of main channel radiances. Note that the variation in spectral co-registration shows some correlation with the relative change in temperature between PMD-P and PMD-S, however only a very small relative change has been observed which is negligible with respect to accuracy requirements for " $q$ " Stokes fraction derivations.

Another consequence of the lack of thermal stabilization of the optical bench is that a strong coupling, on all timescales (long-term, seasonal, and orbital), has been observed between the full width half maximum (FWHM) of the instrument spectral response function and the optical bench temperature. It is understood that the sensitivity of the FWHM to the optical bench temperature, and also temperature gradients within the instrument, is amplified due to the defocusing of the instrument which was performed during the on-ground development with the aim of increasing the spectral oversampling. Figure 10 shows the variation of the FWHM derived from a set of distinct SLS lines which are well separated from their neighbours, allowing for a stable Gaussian shape to be fitted. Note that the Gaussian shape applied in the analysis is normal, and no distortion is applied, although the true slit-function shape is known to be asymmetrical and that the degree of asymmetry varies with wavelength. Nevertheless, in the absence of instability, the derived FWHM should also be stable. However, it has been observed that this is not the case and that the change in FWHM follows two patterns.

First, a spectrally well-ordered pattern (see Fig. 10) during which the FWHM continuously decreases especially for the lower wavelength range is evident. A similar pattern has been observed in the solar Fraunhofer lines by users of level 1 data. This long-term change is anti-correlated with the long-term trends in the optical bench temperature of the instrument (see Fig. 11).

Second, the FWHM varies significantly with the seasonal in-orbit change in the thermal environment. This can easily be verified when comparing the seasonal signals with the optical bench temperature provided in Fig. 11. The seasonal signal of FWHM changes is correlated with the optical bench 


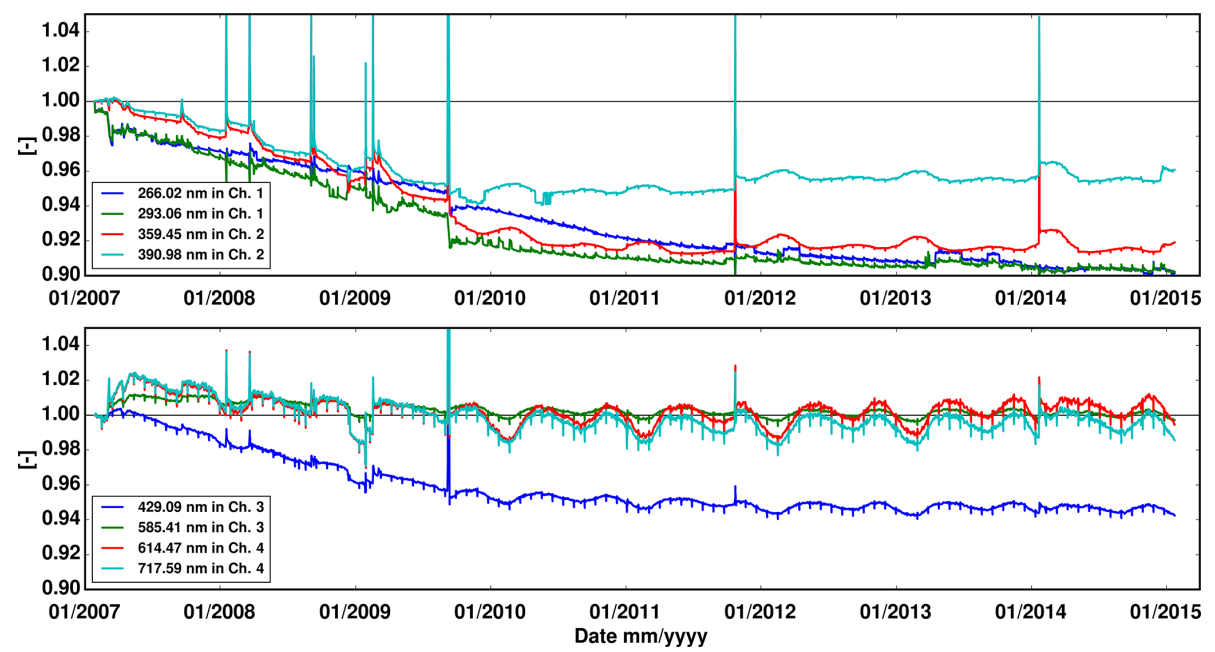

Figure 10. FWHM relative change with respect to January 2007 evaluated from a regular Gaussian fitting of well-separated SLS lines. The upper panel shows the results in channel 1 and 2 . The lower panel results for channel 3 and 4 .

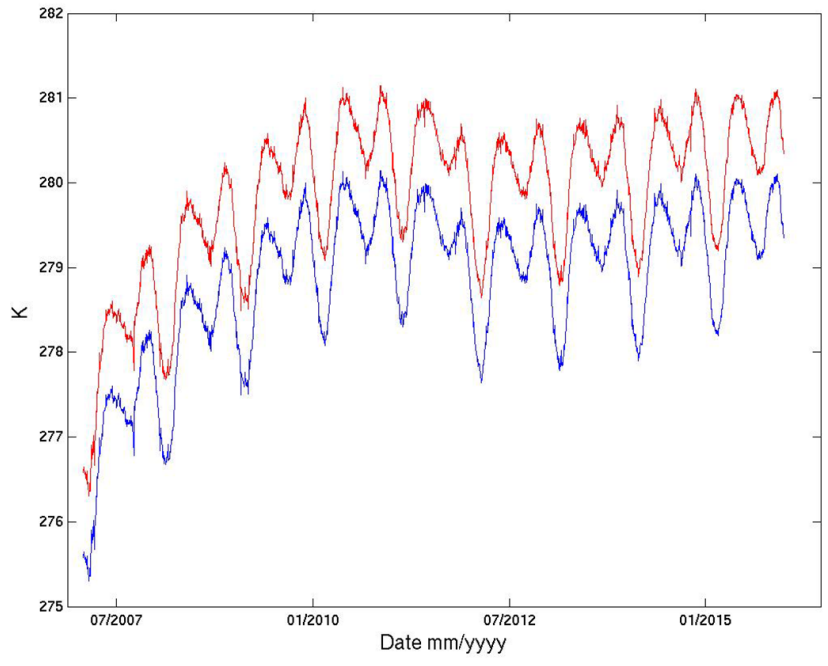

Figure 11. Instrument optical bench temperature sampled once per day in the coldest part of the orbit during solar calibration measurements (blue line) and the maximum temperature encountered during the orbit (red line). The orbital variation (distance between blue and red lines) is about $1 \mathrm{~K}$. All remaining variation is the result of seasonal and long-term changes in platform and therefore instrument temperature.

temperature for channel 1,3 , and 4 , while in channel 2 it is anti-correlated.

Changes in spectral calibration and slit-function shape due to inhomogeneous slit filling are not considered to be an issue due to the averaging effect caused by across-track scanning; however, potential improvements to the spectral calibration by the use of Fraunhofer lines in the solar and/or Earth measurements to account for orbital variation in spectral calibration (van Geffen, 2004) are under evaluation.

\subsection{Etalon correction}

Interference in the thin detector coating layer causes a wavelike pattern on the radiance response (fixed etalon). When deposits settle on the detector coating, the interference pattern is changed (variable etalon). The etalon correction accounts for changes in the variable etalon between on-ground calibration (reference etalon) and the in-orbit situation. It is calculated from daily preprocessed spectra of the on-board WLS. The in-orbit WLS spectrum is nominally ratioed to a reference WLS spectrum representative of the on-ground calibration of the radiance response. The WLS spectra measured in-orbit show an expected characteristic baseline shift as compared to on-ground measurements, which is removed in the data processing. For each channel, a band-pass filter is applied to the ratio. Spectral components within the bandpass (typically 4-10 oscillations per channel) are declared to be the etalon correction. Etalon correction typically is of the order of $10^{-2}$ relative. The remainder is called etalon residual. Applying the etalon correction means dividing a spectrum by the etalon correction spectrum.

As the basis for the etalon correction is a baseline removed ratio of the on-ground reference WLS to the in-orbit lamp measurements, there is an implicit assumption that the channel overlap points, and hence the relative radiometric response in the channel overlap regions has not changed. However, as a result of a significant shift of the channel 1-2 and channel 2-3 overlap points from the on-ground to in-orbit situation (see Sect. 3.10), it was necessary to correct the radiometric key data using in-orbit WLS measurements from the beginning of in-orbit life of the instrument. This correction to the radiometric key data includes by design an implicit etalon correction appropriate to the beginning of life. Transient changes in etalon with respect to the beginning of life situation are now corrected using this in-orbit WLS as a refer- 
Table 7. Validity region of the etalon correction.

\begin{tabular}{lrr}
\hline $\begin{array}{l}\text { Channel } \\
\text { number }\end{array}$ & $\begin{array}{r}\text { Detector pixel } \\
\text { start/stop }\end{array}$ & $\begin{array}{r}\text { Approximate wavelength } \\
\text { start/stop (nm) }\end{array}$ \\
\hline 1 & $310 / 935$ & $243.4 / 312.8$ \\
2 & $210 / 850$ & $316.5 / 392.1$ \\
3 & $120 / 1009$ & $417.1 / 604.1$ \\
4 & $85 / 989$ & $603.2 / 790.8$ \\
PMD-P & $750 / 997$ & $299.9 / 842.3$ \\
PMD-S & $750 / 998$ & $299.9 / 852.3$ \\
\hline
\end{tabular}

ence. In addition, changes in the overlap point due to thermal changes could affect the results. A valid etalon correction is therefore only available in the regions given in Table 7. A closed-loop correction of etalon in the WLS measurements themselves indicates that the etalon is well removed; however, the effect of transient changes in etalon between WLS measurements is difficult to quantify directly from the level 1 data (EUMETSAT, 2013).

\subsection{Sun mean reference (SMR) spectrum}

A calibrated solar spectrum is calculated from preprocessed spectra acquired in sun mode. Spectra within the central part of the sun field of view are absolutely radiometrically calibrated taking solar elevation and azimuth angles for each spectrum into account, and then averaging into a sun mean reference (SMR) spectrum. The radiometric calibration is performed for the spectra as measured, i.e. SMR intensities are not normalized to an Earth-sun distance of $1 \mathrm{AU}$. The SMR spectrum is also spectrally calibrated, correcting the Doppler shift due to the movement of the satellite towards the sun. Applying the SMR spectrum means dividing an Earthradiance spectrum by the SMR spectrum. The SMR irradiance is of the order of $5 \times 10^{14}$ photons $/\left(\mathrm{s} \mathrm{cm}^{2} \mathrm{~nm}\right)$ at $550 \mathrm{~nm}$.

The optical path for the solar irradiance and on-board calibration measurements, taken once per day or during the monthly calibration sequence, is largely similar to the path for the Earth radiance measurements. However, additional optical elements do exist in the optical path for solar and calibration measurements, before the Earth radiance optical path is joined at the scan mirror. Specifically, the solar irradiance reference measurements taken once per day are reflected by a solar diffuser before passing through the calibration unit and arriving at the scan mirror. For a detailed schematic lay-out, see Fig. 2. Most geophysical products are derived from the measured reflectance, the ratio of an Earth radiance measurement to the solar reference spectrum. It is normally assumed that although the optical paths of the solar and Earth radiance measurements are not identical, the influence of the additional optical elements in the solar path is removed by the calibration during the level 0 to 1 data processing. This can however, only be successful if the characteristics of the solar diffuser, in particular the bidirectional scattering distribution function (BSDF), are well characterized on-ground and if optical components do not change during the lifetime of the mission. Any inadequacies in the characterization of the diffuser BSDF on-ground, changes in the diffuser characteristics on-ground to in-orbit, or during the mission due to diffuser degradation, will lead to a degradation of the quality of the reflectance measured. Mischaracterization of the BSDF in elevation direction (with the sun passing through the slit at an approximately fixed azimuth angle during one solar measurement sequence - usually of the order of a few minutes) can lead to a bias introduced in the averaged sun mean reference (SMR) spectrum at any given wavelength. Mischaracterization of the BSDF in azimuth direction will lead to recurring seasonal biases. Mischaracterization in the spectral domain will lead to interferences with spectral absorption features, therefore introducing biases in trace gas column and profile retrievals. In order to verify and ideally correct for such mischaracterizations, analyses have been carried out using in-flight data from various instruments (Slijkhuis, 2005 and references therein). In this paper the in-flight-derived BSDF for the first GOME-2 instrument on Metop-A, in orbit since October 2006, is presented. A reference period of 1 year from August 2011 to July 2012, covering all solar azimuth angles, has been chosen from a relatively late stage of the mission. The reason for choosing this later period is that after more than 3 years in orbit, the instrument has been significantly more stable in terms of degradation as compared to the early years in operations (see Sect. 4), and also mission outages in the later years have been less frequent (EUMETSAT, 2012).

To derive the in-flight BSDF, measurements for all elevation angles (between -1.5 and $1.5^{\circ}$ from the centre of the slit) and all solar azimuth angles acquired over the year (between 317 and $333^{\circ}$ ) are accumulated. They are then sorted and normalized with respect to the geometric centre at elevation angle $0^{\circ}$ and azimuth angle $325^{\circ}$. In order to arrive at the final irradiance Müller matrix element (MMEirr) needed to carry out the radiometric calibration of irradiance signals the derived BSDF is then multiplied by the on-groundcharacterized irradiance response function for each channel. Figure 12 shows the in-flight derived and on-ground characterized MMEirr at $330 \mathrm{~nm}$ including dependencies in both elevation and azimuth direction. Note that the on-ground data have much lower resolution in both elevation $\left(0.75^{\circ}\right.$ for on-ground $0.1^{\circ}$ for in-flight data) and azimuth $\left(2.0^{\circ}\right.$ for onground and $0.5^{\circ}$ for in-flight data) angles. The low resolution of the on-ground characterization in elevation angle cannot capture the observed sinusoidal variation which is likely introduced by shadowing effects of a mesh placed in front of the diffuser in order to attenuate the in-coming flux. Different behaviour is also observed in the azimuth angle domain, particularly for the highest and lowest azimuth angles. These differences are responsible for recurrent patterns seen over a number of years and at many wavelengths in the solar irradiance data time series. The impact of using in-flight-derived 

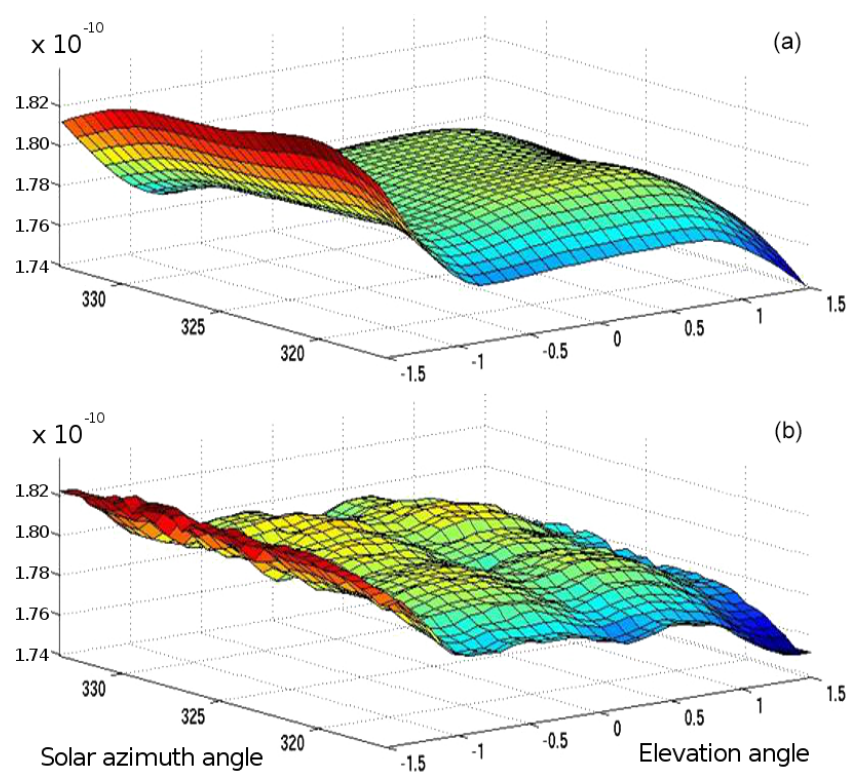

Figure 12. Müller matrix elements (MMEs) at $330 \mathrm{~nm}$ for the radiometric calibration of the irradiance signals including the dependence on elevation and solar azimuth angles. The irradiance response MMEs consist of the diffuser BSDF and the radiometric response per channel. The top panel (a) shows the MME as derived from the on-ground characterization and the lower panel (b) the same but derived from in-flight data.

calibration data in the level 0 to 1 processing, on level 2 data products, is being carefully analysed. Assuming no negative impact, the intention is to use the in-flight-derived data in routine operations.

\subsection{Polarization correction}

GOME-2 is a polarization-sensitive instrument. As such the measured signal depends on the polarization state of the incoming light which in turn depends on the observed scene, in particular observation geometry and cloudiness. The intensity and polarization state of the incoming light is expressed in terms of a Stokes vector, $\boldsymbol{I}=(\mathrm{IQUV}) \equiv \boldsymbol{I}(1$, quv $)$. The Stokes elements IQUV have the dimension of an intensity. The Stokes fractions $q \equiv Q / I, u \equiv U / I, v \equiv V / I$ are dimensionless and assume values between -1 and +1 . The polarization state is characterized by Stokes fractions $q$ (for the $s / p$ component) and $u$ (for the $+45^{\circ} /-45^{\circ}$ component). For each ground pixel, the $q$ Stokes fractions are determined for a number of wavelengths from the observation geometry (single scattering value for the short wavelength end) and ratios of PMD-S and PMD-P band measurements for which there are eight read-outs per main channel read-out. A theoretical estimate of the $u$ Stokes fraction, to which the instrument is less sensitive but which cannot be neglected, is also derived. Stokes fractions are then interpolated to the wavelengths of the FPA detector pixels, and for each pixel a polar- ization correction factor is calculated from Stokes fractions and polarization Müller matrix elements (MMEs) which describe the polarization sensitivity of the instrument. Main channel signals are polarization-corrected by dividing them by the polarization correction factor. The polarization correction is typically of the order of $10^{-2}$ to $10^{-1}$ relative, but may be larger for strongly polarized scenes. Note that it is not possible to apply a polarization correction to the main science channel data from the monthly PMD RAW mode orbit. The quality of this correction mainly depends on the quality of the derived $q$ Stokes fraction and the instrument calibration key-data provided prior to launch. In recent years $q$ Stokes fractions have also been used more frequently for direct retrievals of atmospheric parameters sensitive to the state of polarization of scattered light (e.g. aerosol and cloud parameters).

Due to in-orbit degradation of the GOME-2 instrument (see Sect. 4), which causes loss of throughput in all GOME-2 channels including the PMDs, it is necessary to account for the relative degradation of PMD-P and PMD-S by calculating and applying an update to the relative radiometric response of the PMDs using in-flight PMD band data. The Stokes fraction $q$ depends on the degree of linear polarization $P$ and the polarization angle with respect to the reference plane $\chi$ in the form $q=P \cdot \cos 2 \chi$. Assuming that the polarization angle at all wavelengths is similar to its single scattering value, $\chi_{\mathrm{ss}}$, then $q=0$ when $\cos 2 \chi_{\mathrm{ss}}=0$ independent of the degree of linear polarization, $P$, and regardless of the actual atmospheric scene observed (Schutgens et al., 2004). Therefore, specific locations can be found, taking into account the illumination geometry, where the Stokes fraction $q$ of the light reflected by the Earth's atmosphere is expected to be exactly zero and the ratio of calibrated PMD-P to PMD-S signals for this unpolarized light is expected to be 1 . Any deviation reflects a change in the relative radiometric response of the PMDs. By accumulating data for these special geometries over time, to minimize noise, a correction to the relative radiometric response of the PMDs is derived and subsequently used in the data processing. The update frequency depends on the quality of the accumulated data and the derived correction and is typically between 4 and 7 days. For more details see EUMETSAT (2014b). Subsequent analysis of the calculated $q$ Stokes fractions including application of the online correction, derived from the special geometry conditions described above, serves as a check for the overall consistency of the polarization correction and shows very small deviations (less than 0.02) from zero over the whole time period of the mission. A further analysis of the quality of the polarization measurements from GOME-2 can be found in Tilstra et al. (2014). It should be noted that the residual error in the GOME-2 level 1 products depends not only on the quality of the polarization measurements themselves, but also on the state of polarization of the atmosphere and the calibration key data which characterize the polarization sensitivity of the GOME-2 instrument. 
In order to monitor and validate the measured GOME-2 Stokes fractions for conditions other than the special geometry conditions, a more general approach is needed. The approach used is based on a statistical analysis developed by SRON under contract to ESA (Tilstra, 2008). It can be shown that the general behaviour of the Stokes fraction, $q$, along the orbit is primarily determined by molecular (Rayleigh) scattering, in particular over dark ocean surfaces, and that variability in $q$ is caused by the presence of clouds and aerosols. It is observed that the measured polarization values are always clearly between extreme limiting values. These limiting values lie between the Rayleigh single scattering values and $q=0$. Furthermore, for a large number of measurements the measured polarization values are influenced by largely cloudy scenes which depolarize the light leading to a measured Stokes fraction of $q=0$. The assumption, upon which the generalized validation of $q$ is based, is that the minimum Stokes fractions observed are representative of a limiting atmosphere with minimum depolarization, i.e. a combination of minimum ground albedo and minimum aerosol loading. In the case of little or no instrument degradation these limiting values will be constant in time and can be used as an empirical validation method for the long-term in-flight monitoring of polarization measurements. Figure 13 shows Stokes fractions calculated from Earth radiance scanning measurements as compared to the single scattering Stokes fractions (diagonal line) and $q=0$. Red points lie inside the physically reasonable range, while blue points lie outside the physically reasonable range.

The "limiting atmosphere" plots for $q$ Stokes fraction values are derived for all PMD band values and for one orbit in 2009 (Fig. 13). From the absence of outliers we conclude that the quality of the Stokes fractions and their stability is very good based both on the limiting atmospheres and special geometries analysis. See also Tilstra et al. (2014) for an independent analysis.

\subsection{Stray light correction}

Spectral stray light is (unwanted) light from wavelengths other than the nominal wavelength measured by a given detector pixel. Two types of stray light can be distinguished - uniform and ghost stray light. Uniform stray light originates in diffuse scatter inside the instrument and generates a slowly varying or nearly uniform stray light across a detector array, and ghost stray light originates in specular reflection from optical components within the instrument. For each ground pixel the uniform stray light contribution is calculated by weighting the sum of the measured signals in a channel with the corresponding stray light fraction from the calibration key data. The ghost stray light position and intensity is calculated from the "parent" position and intensity using polynomial coefficients from the key data. Both types have been characterized during on-ground calibration from which it is clear that the most important component is the uniform stray light, particularly in channel 1. Applying the stray light correction means subtracting the total stray light (sum of uniform and ghost stray light) from the measured signals. The stray light correction is typically smaller than $10^{-3}$ relative.

The stray light correction applied during the level 0 to 1 processing uses stray light characterization as delivered following the on-ground calibration and characterization activities. In channel 1 uniform stray light was estimated to be $10 \%$ of the total signal measured in the channel with the relative contribution to the signal increasing at shorter wavelengths as a result of the large dynamic range in the channel. However, several users of the level 1 data have indicated that during retrieval of geophysical products, increasing fitresidual biases have been observed (particularly in channel one and increasing with decreasing wavelength) which may be due to increasing stray light levels (Cai et al., 2012). This is currently under investigation.

\subsection{Calculation of cloud parameters}

An effective cloud fraction and cloud top pressure are retrieved for each GOME-2 ground pixel using the Fast Retrieval Scheme for Clouds from the Oxygen A band (FRESCO+) developed by KNMI (Wang et al., 2008). The FRESCO+ retrieval method is based on a comparison of measured and simulated reflectivities in three approximately $1 \mathrm{~nm}$ wide spectral windows in and around the oxygen $\mathrm{A}$ band $(758,761,765 \mathrm{~nm})$. Information on cloud fraction mainly comes from the continuum (high intensity for high cloud cover). Information on cloud top pressure mainly comes from the oxygen A band itself (high absorption for low cloud). For a detailed validation of the FRESCO+ output in the level $1 \mathrm{~b}$ product we refer to Tuinder et al. (2011).

Additional cloud information, based on the AVHRR cloud mask, is also provided in the GOME-2 level $1 \mathrm{~b}$ product. This comprises geometric cloud fraction and scene homogeneity information derived from AVHRR cloud information and radiance measurements, on a fixed PMD read-out grid of 256 read-outs per scan. Note that in order to use the AVHRR cloud information provided at PMD resolution, for derivation of geophysical products at main channel resolution, it is necessary to carefully account for the synchronization of main channel and PMD data as described in Sect. 3.1. For further information on the AVHRR-derived cloud information please see EUMETSAT (2014e).

Cloud parameters derived both using the FRESCO+ algorithm and from AVHRR data are reported in the GOME-2 level $1 \mathrm{~b}$ products for use in higher level processing (EUMETSAT, 2010, 2014d). They are not used any further in the level 0 to 1 processor.

Figure 14 shows an example of the main channel cloud fraction values from the operational Metop-A FRESCO+ product at $40 \times 40 \mathrm{~km}$ main channel spatial resolution in comparison to the AVHRR-derived geometric cloud fraction 

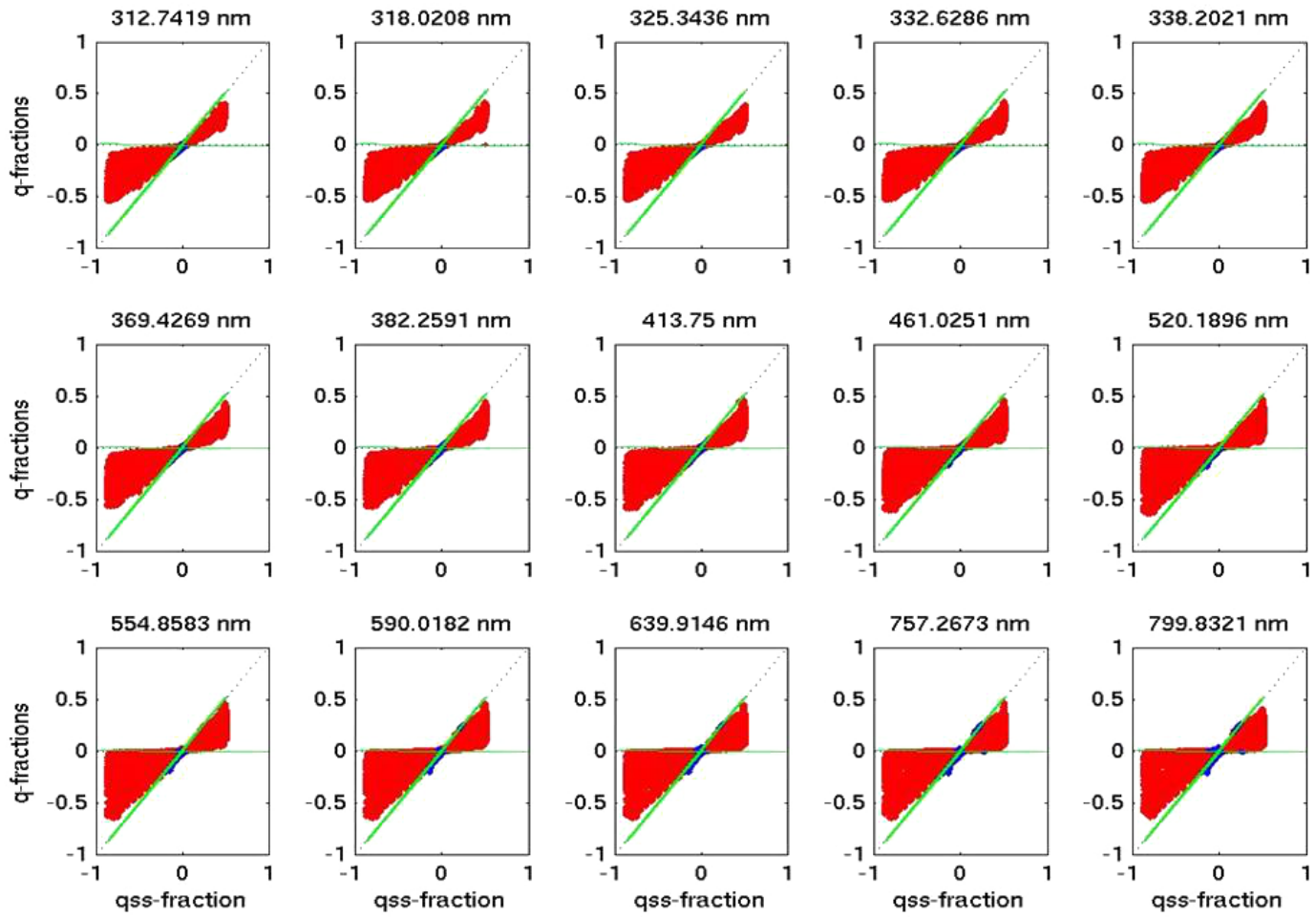

Figure 13. Limiting atmosphere plot for Earth radiance $q$ Stokes fractions and for all PMD bands. The data are derived from one orbit on 31 July 2009. The green lines indicate the Rayleigh single scattering values and $q=0$. Red points lie inside the physically reasonable range, while blue points lie outside the physically reasonable range.

at PMD resolution $(5 \times 40 \mathrm{~km})$. In addition the FRESCO+ cloud top height (at main channel resolution) and the AVHRR derived scene homogeneity (at PMD resolution) are also presented.

The results show the fundamental difference between radiometric cloud fraction values (FRESCO+) and geometric cloud fraction values as derived from AVHRR imager data. Optically thin clouds have significantly less impact or are not accounted for at all in radiometric cloud fraction as, for the radiometric cloud fraction, there is a strong relation between optical thickness and cloud fraction. The geometric cloud fraction is however much more sensitive to the presence of a cloud. Please note however, that depending on the application and the radiative transfer model used for a given geophysical parameter retrieval, the radiometric cloud fraction could be the more appropriate choice since not all retrieval algorithms rely on an accurate and very strict cloud screening. The ones that do may favour the geometric cloud fraction instead. For more details on this issue we refer to Loyola et al. (2007), Tuinder et al. (2011), and EUMETSAT (2014e).

\subsection{Application of radiance response}

Absolutely calibrated radiances in physical units (photons/( $\left.\mathrm{s} \mathrm{cm}^{2} \mathrm{~nm} \mathrm{sr}\right)$ ) are calculated from preprocessed signals in instrument units $\left(\mathrm{BU} \mathrm{s}^{-1}\right)$. This is done by dividing the preprocessed signals by the radiance response MME for the actual scanner viewing angle.

Radiances are strongly dependent on observation geometry and ground scene. Even for a fixed geometry a variation of 1 order of magnitude between a cloudy and a cloud-free scene is common. For a solar zenith angle of $60^{\circ}$, typical radiance values are between $10^{13}$ and $10^{14}$ photons $/\left(\mathrm{s} \mathrm{cm}^{2} \mathrm{~nm} \mathrm{sr}\right)$ in the visible to near-infrared spectrum.

The point where the radiometric response of the instrument is equal for two physically separate detector arrays (channels) is called the overlap point between channels. The spectra per channel are usually cut at this overlap point and concatenated when radiances are to be used from spectral regions bridging a channel separation. This commonly happens for the channel 1 and 2 overlap point around $311 \mathrm{~nm}$. Many level 2 retrievals involving ozone and $\mathrm{SO}_{2}$ use radiances from both above and below this separation point. In addition, many radiometric corrections evaluated in this region are carried out only within the spectral region defined by the overlap points between channels to avoid regions of low signal-to-noise ratio and to guarantee a homogenous transition from one channel to the other.

It has been observed that the channel 1-2 and channel 2-3 overlap points shift significantly from the on-ground to inorbit situation for all instruments, and furthermore that the overlap points are not stable in wavelength but also shift in- 

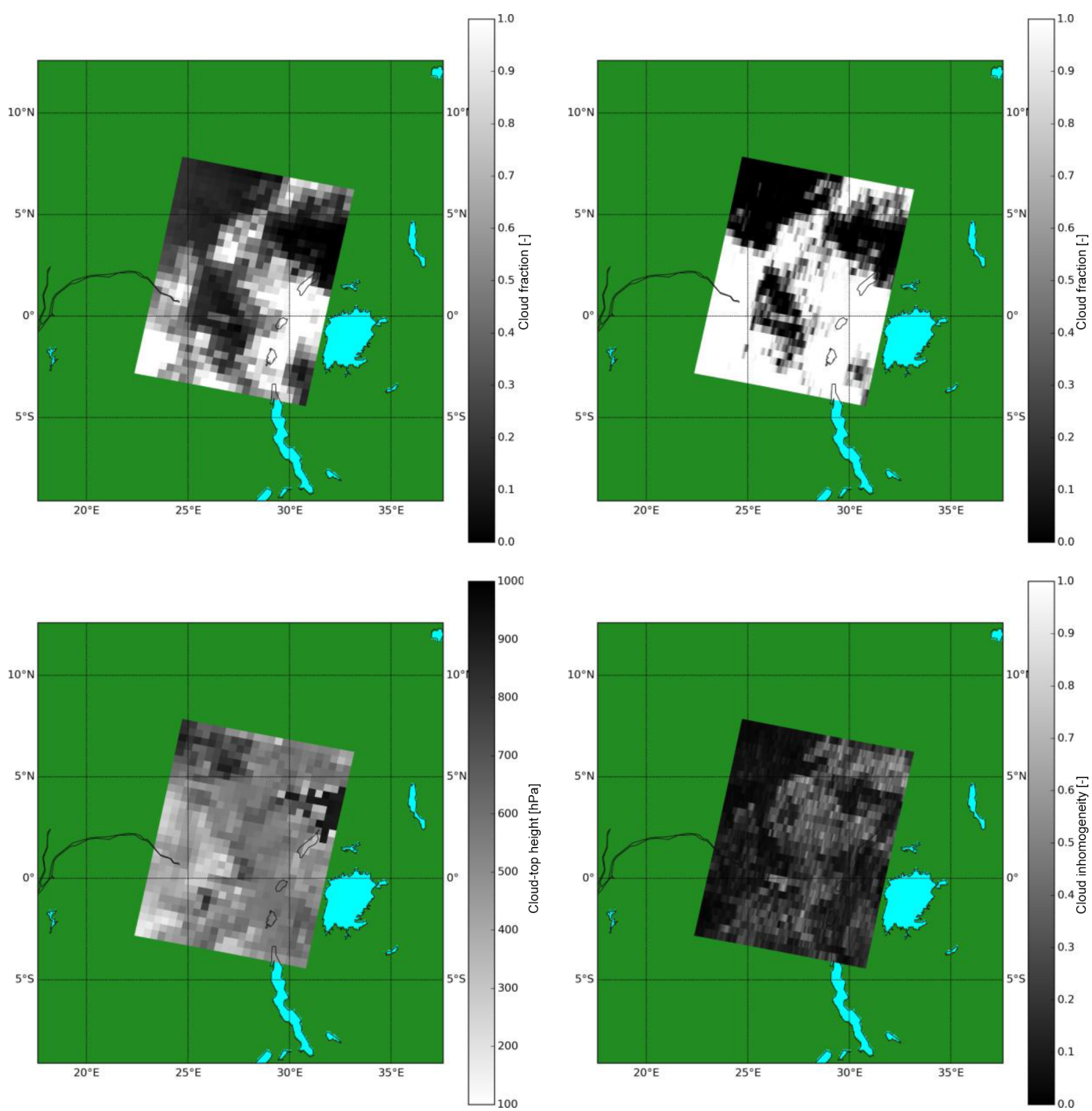

Figure 14. FRESCO+ effective (or radiometric) cloud fraction (upper left panel) and the AVHRR-derived cloud fraction (upper right panel) for sample data for GOME-2 Metop-A from March 2015. Also shown are the FRESCO+ cloud top height (lower left panel) and the AVHRRderived inhomogeneity (lower right panel).

orbit. These changes are assumed to be due to changes in thermal stress on the channel separator prism which controls the channel $1-2$ and $2-3$ separation (see Fig. 2). The channel 2-3 overlap point is more affected than the channel 1-2 overlap point; however, the region between 395 and $405 \mathrm{~nm}$ is rarely used for level 2 retrievals. The channel 1-2 overlap point again shifted significantly following the second throughput test in September 2009 (see EUMETSAT, 2012 for more details). The shift was on the order of $0.1 \mathrm{~nm}$ and had some affect on the etalon correction (see Sect. 3.5). Thereafter, the overlap point gradually shifted back towards $311.5 \mathrm{~nm}$ - the position before the second throughput test.

\subsection{Application of irradiance response}

Absolutely calibrated irradiances in physical units (photons/( $\left.\mathrm{s} \mathrm{cm}^{2} \mathrm{~nm}\right)$ ) are calculated from preprocessed signals in instrument units $\left(\mathrm{BU} \mathrm{s}^{-1}\right)$. This is done by dividing the preprocessed signals by the irradiance response MME for the actual solar elevation and azimuth angle combination appropriate to the measurement. As stated above for the SMR, the solar irradiance is of the order of $5 \times 10^{14}$ photons $/\left(\mathrm{s} \mathrm{cm}^{2} \mathrm{~nm}\right)$ at $550 \mathrm{~nm}$. 


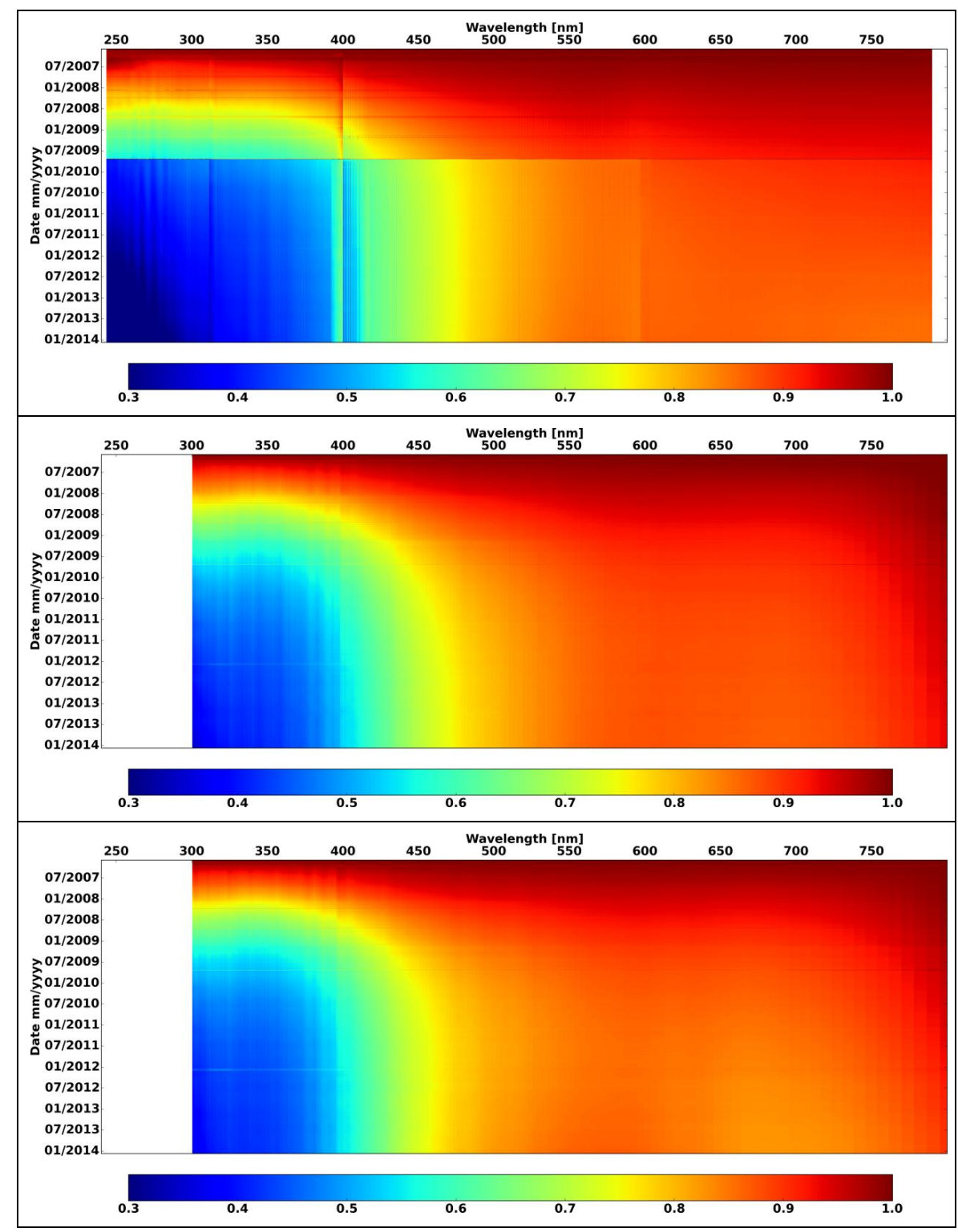

Figure 15. SMR spectra for all four main channels (upper panel), PMD-S (middle panel), and PMD-P (lower panel) normalized to January 2007. The discontinuity in the main channels in September 2009 is the second throughput test (EUMETSAT, 2011). The discontinuities in spectral space for the main channels are the channel overlap regions.

\section{Level 1b data record consistency and instrument degradation}

The long-term consistency of the level $1 \mathrm{~b}$ data set is determined by short, medium, and long-term changes at processor and at instrument level, most notably the instrument throughput. The impact on the consistency of the data set due to changes in the level 0 to 1 processor quality is essentially removed by reprocessing of the data set (see EUMETSAT, 2012). However, short- to long-term changes at instrument level due to instrument anomalies and instrument degradation can only be mitigated by soft corrections which have to be based on evaluation of a consistent data set. Short- to long-term changes in instrument throughput may affect the geophysical parameter retrieval quality in two ways: first, in the case where the signal-to-noise limit required for a specific retrieval of geophysical parameters is reached; second, in the case where the differences in throughput degradation for different optical paths (in particular the Earth radiance optical path as compared to the solar optical path) alter the derived reflectivity values.

Note that under hypothetical conditions where the solar path throughput and the Earth radiance path throughput would degrade in exactly the same way, the level 2 retrievals 
would not be affected by throughput degradation or a changing instrument, except due to loss of signal-to-noise ratio.

During nominal operations, a solar measurement sequence is carried out daily. From this sequence, a solar mean reference spectrum is derived for use in level 2 retrievals that use reflectivity as input. Since the sun serves as a stable radiometric calibration source, these measurements can be used to monitor the instrument throughput stability. The instrument throughput degradation (defined as the relative change in the calibrated measurement of a stable input source e.g. the sun or WLS, where all components in the optical path can contribute to the degradation) is an important measure of the instrument health and the consistency of the reprocessed data records. Apart from instrument-related features, we expect to see no impact of processor- or calibration-related features in a reprocessed sun mean reference (SMR) spectrum time series. Figure 15 provides an overview of the SMR time series at all wavelengths for the four main channels. In this figure, which is largely intended to be illustrative, features which may be related to solar cycle activity have not been removed; however, when analysing the solar spectra for the purposes of derivation of a degradation correction, such effects and the vertical striping in the 300 to $400 \mathrm{~nm}$ range, the exact origin of which is not known, will both be taken into account. One can clearly see that from early in the life of the GOME2 instrument on Metop-A there has been degradation in the optical path of the instrument for the sun measurements, both main channels and PMDs. Examination of other calibration sources shows a similar pattern. The monitoring of long-term Earth radiance signals is complicated by the influence of constantly changing atmosphere and surface properties. Both of these can be expected to exhibit seasonal and inter-annual variations. Earth radiance data are therefore evaluated only over certain regions of the Sahara, the Pacific Ocean, and ice sheets in the Antarctic and Greenland. These regions are known to provide relatively stable conditions during the year and from year to year, and minimize the effect of seasonality, pollution events, and local climate changes. This analysis also shows similar loss of throughput.

Degradation in GOME-2 most likely has two origins: contamination of the primary scan mirror, the most exposed optical element, which was expected based on GOME/ERS-2 (Coldewey-Egbers et al., 2008) and SCIAMACHY/ENVISAT (Noël et al., 2007) experience, and contamination in the region of the detectors themselves which has not been observed previously in the UV-visible spectral region. The degradation may have different characteristics for different optical paths (e.g. Earth, sun, WLS) depending on the optical components involved. For example analysis of the SLS over diffuser measurements, despite the measurements being very unstable (see Sect. 1.5.2), indicates an upper boundary for diffuser degradation of less than $10 \%$ over 7 years in orbit. In contrast the initial analysis of the differential degradation between the solar and Earth radiance measurements suggests a higher rate of degradation at the lowest wavelengths which may be due to the primary mirror at the sun-port entrance (see inlay A of Fig. 2). Loss of signal necessarily decreases signal-to-noise ratios. Degradation of mirror surfaces may also affect the polarization response leading to an angular dependence in degradation effects (Krijger et al., 2014). Additionally, changes in the characteristics of the diffuser used to view the sun or features in the level $1 b$ data arising from the diffuser can lead to artefacts in the level 2 data products (EUMETSAT, 2011).

Degradation of the GOME-2 instrument on Metop-B has also been observed and is similar but not exactly the same as that observed for GOME-2 on Metop-A with a slightly lower degradation rate in channel 2 (EUMETSAT, 2015a, b).

The impact of GOME-2 degradation on level 2 product quality is highly dependent on the individual level 2 algorithms in question, and can only be meaningfully discussed in parallel with a full level 2 algorithm description. A discussion of the impact of degradation on a range of level 2 products is therefore outside the scope of this paper. However, this subject has been extensively evaluated for selected level 2 products in two studies commissioned by EUMETSAT, the results of which are documented in Dikty (2011) and Azam (2015).

The analysis of the GOME-2 degradation, including an initial evaluation by level 2 product providers, is ongoing for all operational instruments with the aim of characterizing the degradation in reflectivity, in spectral, angular, and temporal space, and to provide a degradation correction to users. Note however that signal-to-noise ratio cannot be recovered. The degradation analysis and correction will be the subject of a dedicated publication.

\section{Conclusions}

The GOME-2 instrument, which flies on the Metop series of satellites, the first of which was launched on 19 October 2006, senses the Earth's backscattered radiance and extraterrestrial solar irradiance in the ultraviolet and visible part of the spectrum. Extensive on-ground calibration and characterization, and in-orbit calibration activities have guaranteed the provision of high-quality calibrated level 1 data which are provided to users in near-real time. Ongoing monitoring and analysis activities guarantee the continued high quality of the level 1 data. Improvements to the characterization of the BSDF of the solar diffuser using in-flight data are in progress, and potential improvements to the spectral calibration using Fraunhofer lines in the solar and/or Earth measurements (van Geffen, 2004) are under consideration. Instrument degradation causing loss of throughput in the instruments and improved stray light correction is the subject of an ongoing analysis which will result in a combined correction to be provided to users. This analysis and a detailed description of individual calibration improvements will be the subject of a separate publication. The operational level 2 
GOME-2 products are produced by the EUMETSAT Satellite Application Facility on Ozone and Atmospheric Chemistry Monitoring and are presented in this special issue by Hassinen et al. (2016).

\section{Data availability}

Note that EUMETSAT technical documents and study reports can be found at www.eumetsat.int $>$ Data $>$ Technical Documents $>$ GDS Metop $>$ GOME-2. Calibration-related documents from TNO Space Systems Engineering and TPD Space Instrumentation are available as part of the calibration key data package from the GOME-2 monitoring pages in the documentation subsection at http://gome.eumetsat.int.

Acknowledgements. The authors would like to acknowledge the work of the large team of colleagues at EUMETSAT, ESA, and in industry, and including our scientific partners, that have contributed to the success of the whole EUMETSAT Polar System, and in particular the GOME-2 instrument.

Edited by: J. Tamminen

\section{References}

Azam, F., Richter, A., Weber, M., Noël, S., and Burrows, J. P.: GOME-2 on MetOp-B Follow-on analysis of GOME2 in orbit degradation, EUM/CO/09/4600000696/RM, Final Report, 2015.

Beirle, S., Sihler, H., and Wagner, T.: Linearization of the effects of spectral shift and stretch in DOAS analysis, Atmos. Meas. Tech., 6, 661-675, doi:10.5194/amt-6-661-2013, 2013.

Cai, Z., Liu, Y., Liu, X., Chance, K., Nowlan, C. R., Lang, R., Munro, R., and Suleiman, R.: Characterization and correction of Global Ozone Monitoring Experiment 2 ultraviolet measurements and application to ozone profile retrievals, J. Geophys. Res., 117, D07305, doi:10.1029/2011JD017096, 2012.

Callies, J., Corpaccioli, E., Eisinger, M., Hahne, A., and Lefebvre, A.: GOME-2 - Metop's Second-Generation Sensor for Operational Ozone Monitoring, ESA Bull.-Eur. Space, 102, 28-36, 2000.

Coldewey-Egbers M., Slijkhuis, S., Aberle, B., and Loyola, D.: Long-term analysis of GOME in-flight calibration parameters and instrument degradation, Appl. Optics, 47, 4749-4761, doi:10.1364/AO.47.004749, 2008.

Dikty, S., Richter, A., Weber, M., Noël, S., Bovensmann, H., Wittrock, F., and Burrows, J. P.: GOME-2 on MetOp-A Support for Analysis of GOME-2 In-Orbit Degradation and Impacts on Level 2 Data Products, ITT 09/10000262, Final Report, 2011.

EUMETSAT: GOME-2 L1 Product Format Specification, EUMETSAT, Darmstadt, Germany, EPS.MIS.SPE.97232, v9, 74 pp., 2010.

EUMETSAT: Investigation on GOME-2 Throughput Degradation, EUMETSAT, Darmstadt, Germany, EUM/LEO/REP/09/0732, Issue 1.1, 58 pp., 2011.

EUMETSAT: GOME-2/Metop-A Level 1B Product Validation Report No. 5: Status at Reprocessing, EUMETSAT, Darm- stadt, Germany, G2RP-R2, EUM/OPS/EPS/REP/09/0619, v1F, 110 pp., 2012.

EUMETSAT: EPS Metop-B Product Validation Report: GOME-2 Level, EUMETSAT, Darmstadt, Germany, EUM/OPS/EPS/DOC/12/0760, 150 pp., 2013.

EUMETSAT: GOME-2 Factsheet, EUMETSAT, Darmstadt, Germany, EUM/OPS/DOC/10/1299, v4b, 33 pp., 2014a.

EUMETSAT: GOME-2 L1 Product Generation Specification, EUMETSAT, Darmstadt, Germany, EPS.SYS.SPE.990011, v8, 462 pp., $2014 b$.

EUMETSAT: GOME-2/Metop-A PMD Band Definitions 3.0 and PMD Calibration, EUMETSAT, Darmstadt, Germany, EUM/OPS/EPS/DOC/12/0714, v1A, 15 pp., 2014c.

EUMETSAT: Generic Product Format Specification (GPFS), EUMETSAT, Darmstadt, Germany, EPS.GGS.SPE.96167, v8B, 72 pp., 2014d.

EUMETSAT: GOME-2 PPF6 AVHRR Cloud Properties Validation Report, EUMETSAT, Darmstadt, Germany, EUM/TSS/DOC/14/763526, v1, 32 pp., 2014e.

EUMETSAT: Metop-A GOME Annual In-Flight Performance Review 2014, EUMETSAT, Darmstadt, Germany, EUM/OPSEPS/REP/14/770718, v2A, 94 pp., 2015a.

EUMETSAT: Metop-B GOME Annual In-Flight Performance Review 2014, EUMETSAT, Darmstadt, Germany, EUM/OPS/EPS/REP/14/771772, v2A, 84 pp., 2015 b.

Hassinen, S., Balis, D., Bauer, H., Begoin, M., Delcloo, A., Eleftheratos, K., Gimeno Garcia, S., Granville, J., Grossi, M., Hao, N., Hedelt, P., Hendrick, F., Hess, M., Heue, K.-P., Hovila, J., JønchSørensen, H., Kalakoski, N., Kauppi, A., Kiemle, S., Kins, L., Koukouli, M. E., Kujanpää, J., Lambert, J.-C., Lang, R., Lerot, C., Loyola, D., Pedergnana, M., Pinardi, G., Romahn, F., van Roozendael, M., Lutz, R., De Smedt, I., Stammes, P., Steinbrecht, W., Tamminen, J., Theys, N., Tilstra, L. G., Tuinder, O. N. E., Valks, P., Zerefos, C., Zimmer, W., and Zyrichidou, I.: Overview of the O3M SAF GOME-2 operational atmospheric composition and UV radiation data products and data availability, Atmos. Meas. Tech., 9, 383-407, doi:10.5194/amt-9-3832016, 2016.

Klaes, D., Cohen, M., Buhler, Y., Schlüssel, P., Munro, R., Luntama, J.-P., von Engeln, A., O'Clerigh, E., Bonekamp, H., Ackermann, J., and Schmetz, J.: An Introduction to the EUMETSAT Polar system, B. Am. Meteorol. Soc., 88, 1085-1096, doi:10.1175/BAMS-88-7-1085, 2007.

Krijger, J. M., Snel, R., van Harten, G., Rietjens, J. H. H., and Aben, I.: Mirror contamination in space I: mirror modelling, Atmos. Meas. Tech., 7, 3387-3398, doi:10.5194/amt-7-3387-2014, 2014.

Loyola, D. W. T., Livschitz, Y., Ruppert, T., Albert, P., and Hollmann, R.: Cloud properties derived from GOME/ERS-2 backscatter data for trace gas retrieval, IEEE T. Geosci. Remote, 45, 2747-2758, 2007.

Munro, R., Eisinger, M., Anderson, C., Callies, J., Corpaccioli, E., Lang, R., Lefebvre, A., Livschitz, Y., Pérez Albiñana, A.: GOME-2 on MetOp, Proc. of the 2006 EUMETSAT Meteorological Satellite Conference, Helsinki, Finland, 12-16 June 2006, EUMETSAT p. 48, 2006.

Noël, S., Bramstedt, K., Bovensmann, H., Burrows, J. P., Gottwald, M., and Krieg, E.: SCIAMACHY Degradation Monitoring Re- 
sults, Proc. of the ENVISAT Symposium, Montreux, Switzerland, 23-27 April 2007, ESA p. 1, 2007.

Schutgens, N. A. J., Tilstra, L. G., Stammes, P., and Bréon, F.-M.: On the relationship between Stokes parameters Q and U of atmospheric ultraviolet/visible/near-infrared radiation, J. Geophys. Res., 109, D09205, doi:10.1029/2003JD004081, 2004.

Slijkhuis, S., Wahl, S., Aberle, B., and Loyola, D.: Re-analysis of GOME/ERS-2 Diffuser properties, Proceedings of the 2004 Envisat and ERS Symposium, 6-10 September 2004, Salzburg, Austria, SP-572 European Space Agency, 2005.

Tilstra, L. G.: GOME-2 Polarisation Study Final Report, SROMEOS-RP-08-033, Issue 0.3, 2008.

Tilstra, L. G., Lang, R., Munro, R., Aben, I., and Stammes, P.: Contiguous polarisation spectra of the Earth from 300 to $850 \mathrm{~nm}$ measured by GOME-2 onboard MetOp-A, Atmos. Meas. Tech., 7, 2047-2059, doi:10.5194/amt-7-2047-2014, 2014.

TNO: Space Systems Engineering: GOME-2 Calibration: Data analysis procedures, MO-RS-TPD-GO-0027, 2011a.

TNO: Space Systems Engineering: GOME2 Calibration: key data file structure specification, MO-RS-TPD-GO-0025, 2011b.
TNO: Space Systems Engineering: GOME-2 Instrument Calibration: key data status list, MO-LI-TPD-GP-0026, 2012.

TPD: Space Instrumentation: GOME-2 Calibration Error Budget, MO-RS-TPD-GO-0016, 2003.

TPD: Space Instrumentation: GOME-2 Calibration Plan, MO-PLTPD-GO-0004, 2004a.

TPD: Space Instrumentation: GOME-2 FM3 Slit Function Test Report MO-TR-TPD-GO-0101, 2004b.

Tuinder, O., Wang, P., and Stammes, P.: Support for upgrade to FRESCO+ in the GOME-2 PPF: Final Report, EUM/CO/09/4600000655/RM, 2011.

van Geffen, J. H. G. M.: Wavelength calibration of spectra measured by the Global Ozone Monitoring Instrument: variation along orbits and in time: Appl. Opt., 43, 695-706, 2004.

Wang, P., Stammes, P., van der A, R., Pinardi, G., and van Roozendael, M.: FRESCO+: an improved $\mathrm{O}_{2}$ A-band cloud retrieval algorithm for tropospheric trace gas retrievals, Atmos. Chem. Phys., 8, 6565-6576, doi:10.5194/acp-8-6565-2008, 2008. 